\title{
REVIEW
}

\section{Graphene membranes for water desalination}

\author{
Shahin Homaeigohar ${ }^{1}$ and Mady Elbahri ${ }^{1,2}$ \\ Extensive environmental pollution caused by worldwide industrialization and population growth has led to a water shortage. \\ This problem lowers the quality of human life and wastes a large amount of money worldwide each year due to the related \\ consequences. One main solution for this challenge is water purification. State-of-the-art water purification necessitates the \\ implementation of novel materials and technologies that are cost and energy efficient. In this regard, graphene nanomaterials, \\ with their unique physicochemical properties, are an optimum choice. These materials offer extraordinarily high surface area, \\ mechanical durability, atomic thickness, nanosized pores and reactivity toward polar and non-polar water pollutants. These \\ characteristics impart high selectivity and water permeability, and thus provide excellent water purification efficiency. This review \\ introduces the potential of graphene membranes for water desalination. Although literature reviews have mostly concerned \\ graphene's capability for the adsorption and photocatalysis of water pollutants, updated knowledge related to its sieving \\ properties is quite limited.
}

NPG Asia Materials (2017) 9, e427; doi:10.1038/am.2017.135; published online 25 August 2017

\section{INTRODUCTION}

Currently, $\sim 1.2$ billion people around the world are suffering from a shortage of water and its adverse consequences on health, food and energy. ${ }^{1,2}$ On one hand, population growth, increased industrialization and greater energy needs and, on the other hand, loss of snowmelt, shrinkage of glaciers and so on will worsen this situation in upcoming years. As estimated by the world water council, the number of affected people will rise to 3.9 billion in the coming decades. ${ }^{2,3}$

One of the most promising approaches to alleviate the water shortage, desalination can increase the water supply beyond what is available from the hydrological cycle. ${ }^{4}$ Seawater desalination indeed provides an infinite, steady supply of high-quality water that does not harm natural freshwater ecosystems.

Seawater comprises a vast supply of water $(97.5 \%$ of all water on the planet). Thus, the growth of the installation of seawater desalination facilities in the past decade to circumvent water shortage problems in water-stressed countries has progressed quickly. In 2016, the global water production by desalination was estimated to be 38 billion cubic meter per year, that is, two times higher than that in $2008 .^{5}$

So far, seawater desalination has been mainly performed via multistage flash distillation and reverse osmosis (RO). ${ }^{6}$ Mostly in the arid Persian Gulf countries, desalination plants perform based on heating and then condensing seawater. This kind of desalination plant consumes large amounts of thermal and electric energy, thus emitting greenhouse gases extensively. ${ }^{7}$ In addition to this non-economical and non-ecofriendly version of desalination plants, the main type of desalination plants constructed in the past two decades, as well as future planned ones, are based on RO technology (Figure 1). ${ }^{8}$
In the RO process, water is forced through a semi-permeable membrane by an external positive hydrostatic pressure. Accordingly, a larger volume of water passes through the membrane compared with the volume of dissolved salts or organic molecules. ${ }^{2}$ Thanks to economical and technical efficiencies regarding the desalination of seawater on a large scale, over $50 \%$ of the entire desalination capacity is supplied by $\mathrm{RO}$ systems. ${ }^{1,2} \mathrm{RO}$ is indeed the most energy-efficient technology for seawater desalination and is the benchmark for the comparison of any new desalination technology. The level of energy consumption has even declined in the past 40 years, ${ }^{8}$ owing to technological improvements in the development of membranes with higher permeability and the installation of energy recovery devices and highly efficient pumps. However, this technology still suffers from low desalination capacity and high capital costs, hampering its broad application in most developing markets. ${ }^{2}$ Thus, research is in progress to overcome such limitations and to create more efficient membranes.

\section{ADVANCED RO MEMBRANES WITH HIGHER WATER PERMEABILITY}

The core of the RO process is a semipermeable membrane that separates pure water from seawater. A membrane is in fact a selective barrier between two homogenous phases. The membrane process divides a feed stream into a retentate and a permeate fragment. Pressure-driven membrane processes, such as RO, employ a pressure gradient between the feed and permeate sides as the driving force of the separation to pass solvent through the membrane. Accordingly, particles and solutes are separated based on size, shape and charge (Figure 2). ${ }^{9}$ Ideally, to achieve the optimum filtration performance, a

\footnotetext{
${ }^{1}$ Department of Chemistry and Materials Science, Nanochemistry and Nanoengineering, School of Chemical Engineering, Aalto University, Aalto, Finland and ${ }^{2}$ Faculty of Engineering, Nanochemistry and Nanoengineering, Institute for Materials Science, Christian-Albrechts-Universität zu Kiel, Kiel, Germany

Correspondence: Dr S Homaeigohar or Professor M Elbahri, Department of Chemistry and Materials Science, Nanochemistry and Nanoengineering, School of Chemical Engineering, Aalto University, Kemistintie 1,C321, Aalto 00076, Finland.

E-mail: mady.elbahri@aalto.fi or shahin.homaeigohar@aalto.fi

Received 3 October 2016; revised 27 February 2017; accepted 30 May 2017
} 


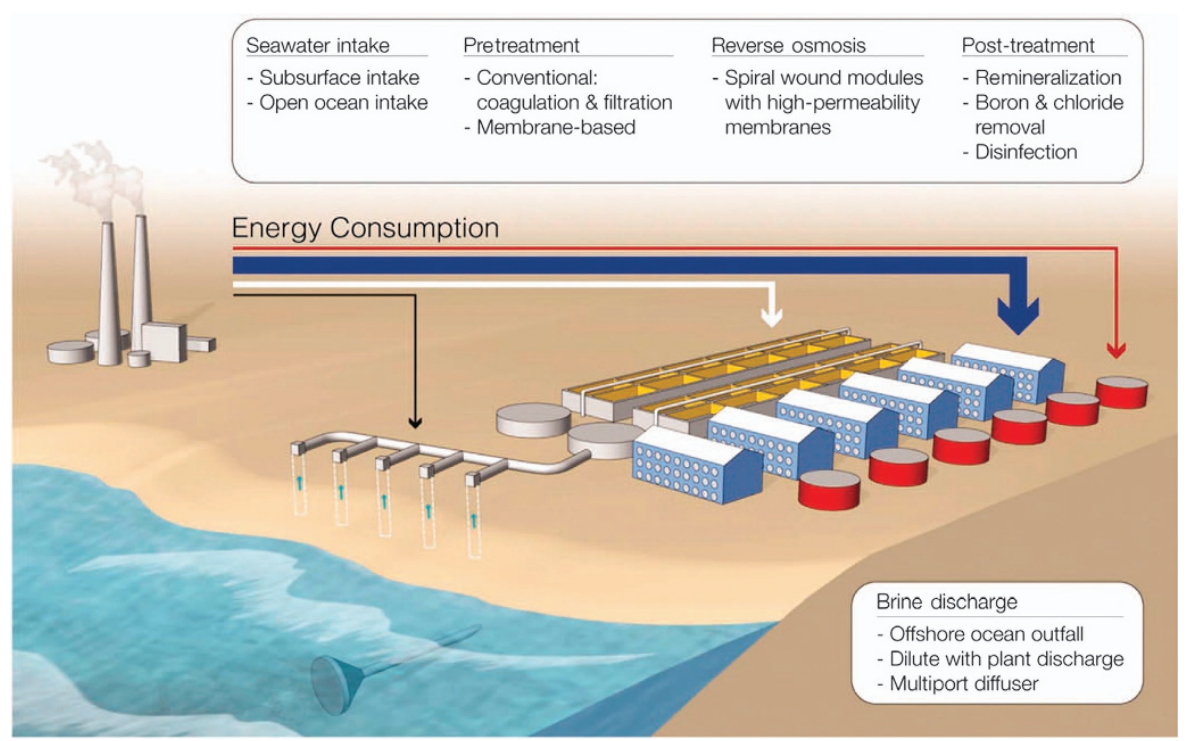

Figure 1 Schematic illustrating a seawater RO desalination plant and its different stages, including seawater intake, pretreatment, reverse osmosis, posttreatment and brine discharge. The arrows represent the energy consumption at each stage proportional to their thickness. (Reprinted with permission from Elimelech and Philip. ${ }^{4}$ Copyright 2016 The American Association for the Advancement of Science.)

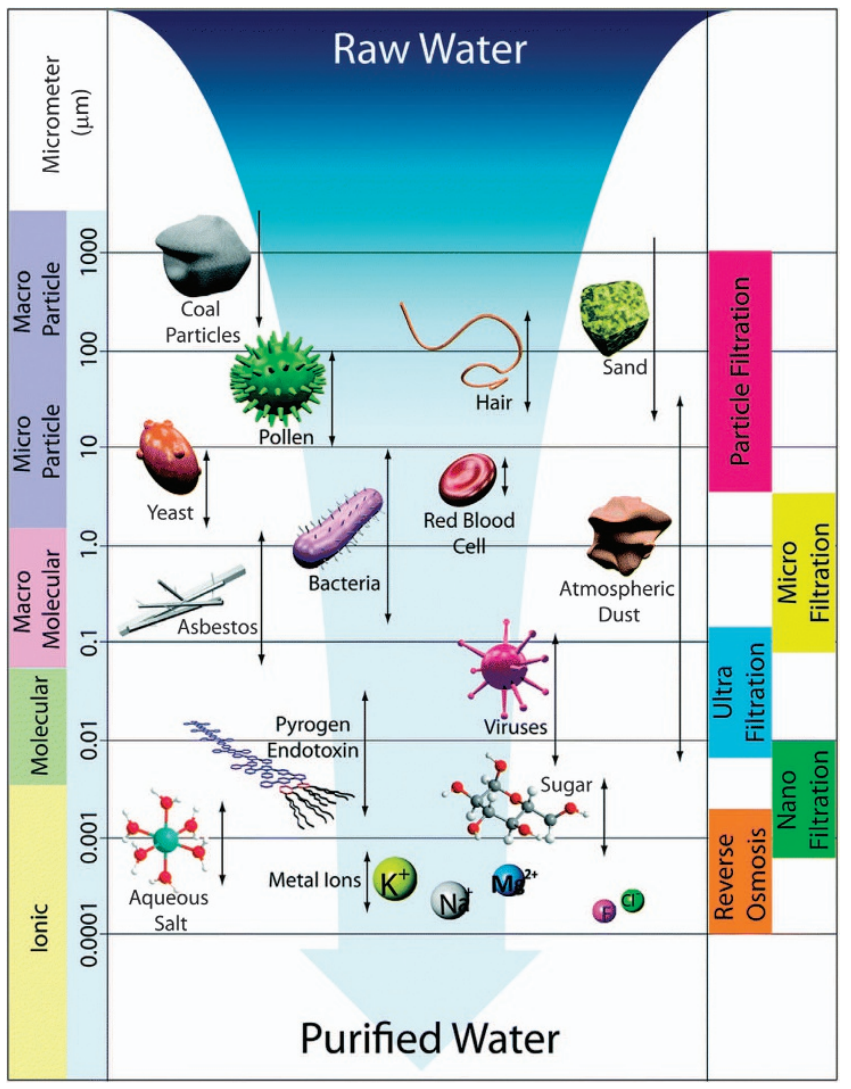

Figure 2 Schematic representation of various membrane processes, including reverse osmosis, NF, ultrafiltration, microfiltration and traditional particle filtration, in which the membrane pore size grows proportionally. RO membranes are in fact dense and non-porous membranes. (Reprinted with permission from Lee et al. ${ }^{85}$ Copyright 2016 Royal Society of Chemistry.)

membrane should be as thin as possible to maximize water permeability, as selective as possible and as mechanically robust as possible to inhibit membrane disintegration. ${ }^{10}$
For the RO desalination process, the development of more permeable membranes can reduce energy consumption and thus related costs. Although the necessary application of pressure in advanced RO systems is nearly equal to the thermodynamic limit, any extra reduction can notably affect the membrane performance. ${ }^{11}$ Cohen-Tanugi and Grossman ${ }^{12}$ found that using an RO membrane with three times higher permeability lowers the required pressure for seawater RO and brackish water plants by $44 \%$ and $63 \%$, respectively. These values can be interpreted as a reduction in energy consumption of 15 and $46 \%$. The reduction in energy consumption is meaningful because of the high cost of energy, that is, $50 \%$ of the total water desalination cost. ${ }^{11}$

The classic semipermeable RO membrane is still based on the same polyamide thin-film composite design that was developed three decades ago. ${ }^{12}$ The most permeable thin-film composite membranes currently offer only 1.5-2 times higher permeability than that 20 years ago and they are still damaged when contacting chlorine; thus, disinfection becomes challenging and the fouling tendency increases. ${ }^{13}$ To meet the needs of advanced desalination required to address the water challenges of the twenty-first century, a breakthrough in RO membrane technology is a must.

Graphene can be regarded as an 'ultimate' RO membrane, because it is stronger, thinner and more chemically resistant than the polyamide active layers in thin-film composite RO membranes. In fact, water flux across a membrane is inversely proportional to its thickness. Thus, the atomic thinness of graphene $(d \approx 0.34 \mathrm{~nm})$ can lead to larger water permeability than the polyamide active layer in thinfilm composite membranes $(d \approx 100 \mathrm{~nm})$. In addition, graphene shows better tolerance to chlorine than polyamide, that is, an important advantage in hindering membrane fouling without degradation. ${ }^{12}$ Graphene as graphene oxide (GO) shows antimicrobial properties, thus lowering membrane biofouling, that is, improving the membrane lifetime and energy consumption of the water purification processes. ${ }^{14,15}$ Moreover, graphene can readily be processed into a membrane for application as RO and nanofiltration (NF) (a low-cost and highly efficient separation technique between ultrafiltration and $\mathrm{RO})$ desalination membranes. For such reasons, extensive research is 
currently being carried out to realize its potential as a next-generation desalination membrane. ${ }^{12}$

At the current primary state, graphene membranes have shown applicability in relevance to water desalinating NF membranes. By themselves, they can desalinate solely mildly brackish water and have shown RO ability via simulation studies as well. For seawater desalination, considering the much larger salt concentration, the membranes need to be significantly modified. This point will be discussed in detail later.

With respect to energy consumption, opposing the previous claim of Cohen-Tanugi and Grossman, ${ }^{12}$ Elimelech and Phillip ${ }^{4}$ state that for seawater RO desalination, the consumed energy is more than the theoretical minimum energy required for desalination. In addition, employing more-permeable membranes, such as those based on carbon nanotubes, aquaporin and graphene, will not significantly reduce this energy level. Such a conflict is mainly due to current RO system designs that desalinate seawater using a single membrane stage fed by a high-pressure pump (supplying a feed pressure of $P_{\mathrm{H}}$ ). Ideally, if the RO system operates at the thermodynamic limit $\left(P_{\mathrm{H}}=\Pi_{\mathrm{c}}\right.$ (osmotic pressure of the concentrate leaving the system), that is, in an ideal system with $100 \%$ efficient pumps and energy recovery devices, and in the absence of concentration polarization or frictional losses down the channel), then a single-stage RO process requires the energy per volume of fresh water produced to be as high as $\Pi_{\mathrm{c}}$. As an example, recovering $50 \%$ of $35 \mathrm{~g} \mathrm{l}^{-1}$ salt-containing seawater necessitates the consumption of a minimum energy of $1.56 \mathrm{kWh} \mathrm{m}^{-3}$ versus the thermodynamic minimum energy of $1.06 \mathrm{kWh} \mathrm{m}^{-3}$. Therefore, $0.50 \mathrm{kWh} \mathrm{m}^{-3}$ is expended due to the system's finite size and its irreversible thermodynamic behavior. Considering the reported energy consumption of $\sim 2 \mathrm{kWh} \mathrm{m}^{-3}$ by well-designed seawater RO systems, such an ideal energy consumption of $1.56 \mathrm{kWh} \mathrm{m}^{-3}$ is not a notable achievement. In a broader perspective, the supplementary steps of pre- and post treatment also add to the total energy consumption of seawater RO systems. Thus, as estimated, the overall energy

a
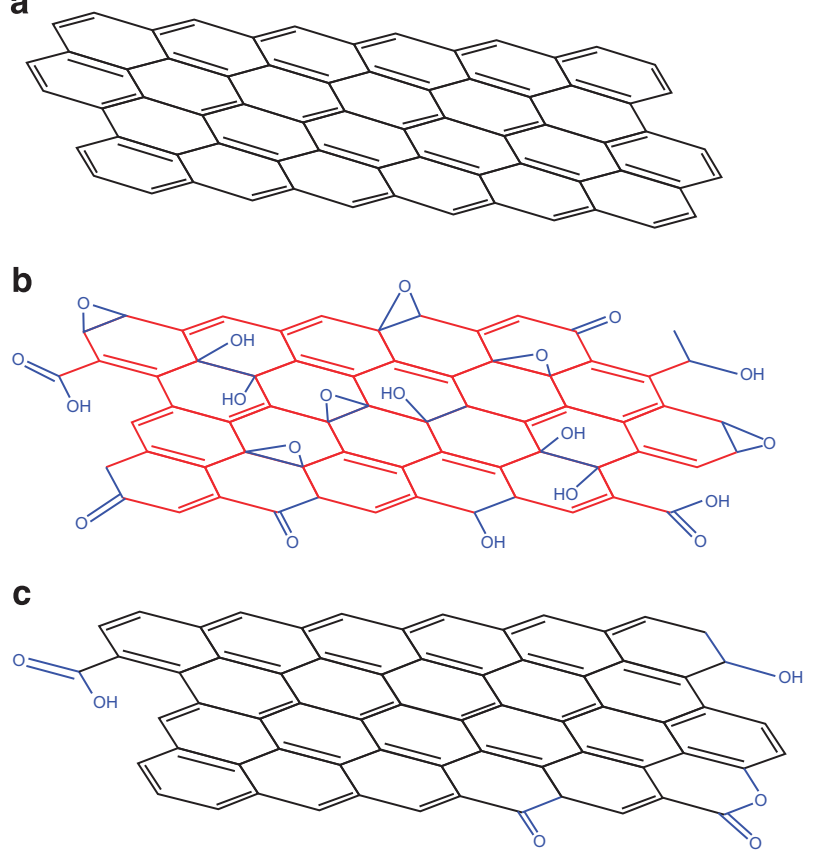

Figure 3 Structural models of single-layer graphene (a), GO (b) and rGO (c). (Reprinted with permission from Zhao et al. ${ }^{86}$ Copyright 2016 American Chemical Society.) consumption is three to four times higher than the theoretical minimum energy. As mentioned earlier, as there is a thermodynamic limit on the energy demand for the desalination step, energy efficiency of the whole desalination cycle should be sought in supplementary processes. For instance, abolishing the pretreatment process would notably lower the energy consumption, capital cost and environmental impact of desalination plants. To achieve this goal, fouling-resistant membranes with tailored surface properties are greatly required. In fact, there is a need for membrane materials with surface chemistries that inhibit the adhesion of diverse foulants, while preserving the high permeability and selectivity necessary for seawater desalination. Graphene materials can have a vital role in this aspect due to their biofouling resistance, hydrophilicity, thinness, functionality and tunable small pore size. Therefore, from this point of view, graphene membranes are promising candidates for next-generation seawater desalination systems.

\section{GRAPHENE NANOMATERIALS FOR WATER PURIFICATION}

Graphene is a two-dimensional (2D) 1-atom-thick planar sheet of $s p^{2}$-bonded carbon atoms. The first synthesis of graphene, using scotch tape and a piece of graphite, dates back to 2004. This material shows amazing physical, mechanical, thermal and optical properties, which have been highlighted in most areas of science and engineering. ${ }^{16}$ The $s p^{2}$ bonds and the involved electron configuration are the main reasons for the ultra-high mechanical strength and elasticity, tunable electronic band gap, excellent thermal $\left(5300 \mathrm{~W} \mathrm{mK}^{-1}\right)$ and electrical conductivity $\left(2000 \mathrm{~S} \mathrm{~cm}^{-1}\right)$ and room-temperature Hall effect of graphene. ${ }^{16-20}$ Its hardness is over 30 times higher than that of diamond and 200 times higher than that of steel. ${ }^{21}$

Regarding water separation, graphene possesses an atomic thickness, assuring its high fluid permeability (several-fold higher than that of most commercial NF membranes) and thus energy/cost efficiency. In addition, there is good potential for size-selective transport through the nanopores of a highly robust graphene layer or $2 \mathrm{D}$ nanochannels between adjacent stacked graphene sheets. The fabrication of graphene-based membranes for desalination is also straightforward. ${ }^{22}$

Graphene can be used for the construction of desalination membranes in various forms, such as pristine graphene, GO and reduced GO (rGO) (Figure 3). Pristine graphene is a single 2D layer of carbon atoms organized in a hexagonal pattern. Layered oxygenated graphene sheets, that is, those including oxygen functional groups, such as epoxides, carboxyls, hydroxyls and alcohols, on their basal planes and edges, are called GO. ${ }^{23,24}$ GO is made via oxidation of natural graphite flakes by using strong oxidants, such as $\mathrm{KMnO}_{4}$, $\mathrm{KClO}_{3}$ or $\mathrm{NaNO}_{2}$, along with a strong acid, for example, concentrated sulfuric acid or nitric acid. Subsequently, single GO sheets can be exfoliated by ultrasonication. A GO sheet is mainly composed of pristine (16\%) and oxidized domains (82\%) with a negligible percentage of holes $(2 \%)$. In this structure, the isolated pristine domains are confined by the continuous oxidized domains. ${ }^{18}$ Through reduction processes, such as chemical reduction, electro-reduction, thermal annealing, flash reduction and enzymatic reduction, GO is converted to rGO with some residual oxygen and structural defects. ${ }^{25}$

\section{GRAPHENE DESALINATION MEMBRANES}

Graphene nanomaterials can be regarded as building blocks of advanced desalination membranes with two main structures: monolayer and stacked multilayer. 


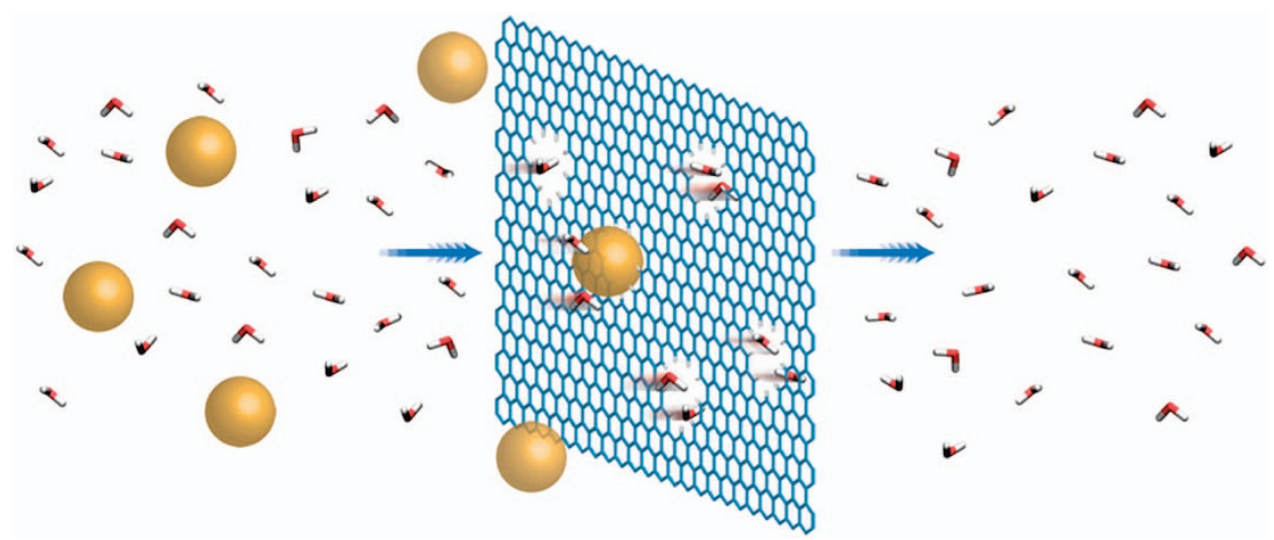

Figure $4 \mathrm{~A}$ graphene membrane with subnanometer pores as an RO membrane. In this process, the salt water (left), subjected to a high pressure, is divided into two parts: water molecules (red and white) passing through the membrane (right) and salt ions (golden spheres) that are blocked. (Reprinted with permission from Wang and Karnik. ${ }^{87}$ Copyright 2016 Nature Publishing Group.)

\section{Monolayer graphene desalination membranes}

Owing to the formation of a dense and delocalized electron cloud from the $\pi$-orbitals of graphene, which blocks the voids within its aromatic rings, pristine graphene is impermeable. Thus, even $\mathrm{He}$ (as the smallest monoatomic molecule with a molecular radius of $1.3 \AA$ ) cannot pass through it. ${ }^{26,27}$ However, according to simulations, by the inclusion of pores of controlled size, density and functionality, graphene membranes can surpass current desalination membranes, showing orders of magnitude higher permeability and selectivity (Figure 4$)^{28}$

Molecular dynamics simulations have predicted that nanoporous graphene, due to its extraordinary water flow rate (up to $661 . \mathrm{cm}^{-2} \cdot \mathrm{day}^{-1} \cdot \mathrm{MPa}^{-1}$ ) and high $(>99 \%)$ salt rejection (depending on the pore size and chemistry), may be one of the most desirable materials for water desalination. ${ }^{2}$ By comparison, a conventional RO membrane can offer same salt rejection efficiency but with a water permeability of only $0.01-0.051 . \mathrm{cm}^{-2}$.day ${ }^{-1} \cdot \mathrm{MPa}^{-1}{ }^{29}$ The cause of the ultrahigh water permeability of graphene, which translates into a notable reduction in the initial capital investment and operating costs of desalination plants, is its atomic thickness. Thus, the creation of controlled pores in terms of size, density and functionality in a graphene structure promises the development of a highly selective and, at the same time, permeable membrane for water desalination. ${ }^{15}$ Such advantages have drawn the attention of researchers to investigate the filtration capabilities of graphene experimentally and through simulations.

Theoretical considerations. The theory for the creation of water purification membranes out of graphene nanomaterials was, for the first time, probed by molecular simulations. ${ }^{2,30}$ In these simulations, nanopores were modeled on the surface of single graphene sheets, permitting water to pass through while selectively rejecting water pollutants. By tuning the pore size and functional groups on the nanopores, theoretically, monolayer graphene can act as a desalination membrane with a water permeability several orders of magnitude higher than that of conventional RO membranes. ${ }^{31}$

Through simulations, Cohen-Tanugi and Grossman ${ }^{30}$ clarified the mechanisms of water transport through a graphene membrane. They showed that hydrogen-functionalized pores facilitate salt rejection but are less water permeable than those functionalized with $\mathrm{OH}$ groups. The reason is that water molecules must be in a specific orientation to pass through a hydrogenated pore. This reorientation carries an entropic penalty, whereby the water permeability declines. On the other hand, both water molecules and solvated salt ions break more hydrogen bonds while passing through the hydrophobic hydrogenated nanopores. In contrast, $\mathrm{OH}$ groups can replace water in the hydration shell of salt ions, thus enabling some ions to permeate, that is, lowering the salt rejection efficiency. Based on simulations, the optimum size enabling the achievement of the highest water permeability for hydrogenated and hydroxylated pores is 23.1 and $16.3 \AA$, respectively. ${ }^{30}$ In addition, the pore density is a determining factor of the water permeability of nanoporous graphene membranes. Suk and Aluru $^{32}$ probed the water permeation mechanisms and hydrodynamic properties of such membranes. They stated that the water flow rate per pore per unit pressure $\left(p_{\mathrm{Q}}\right)$ for a graphene pore is as follows ${ }^{12,32}$

$$
p_{\mathrm{Q}}(a)=\frac{\pi\left(a^{4}+4 a^{3} \delta\right) 1}{8 \mu} L_{\mathrm{h}}
$$

where $L_{\mathrm{h}}$ is the hydrodynamic membrane length, $a$ is the pore radius, $\mu$ is the water viscosity and $\delta$ is the slip length. The equation used for the calculation of $p_{\mathrm{Q}}$ can approximate the water permeability of the membrane, as $\rho p_{\mathrm{Q}}$, where $\rho$ is the number density of nanopores that are water permeable per $\mathrm{m}^{2}$ of the membrane. As a result, the water permeability is dependent on the density of nanopores per unit area. The estimated nanopore density of a nanoporous graphene membrane can be in the range of $10^{11}-10^{13} \mathrm{~cm}^{-2}$, considering a relatively close spacing of nanopores. ${ }^{12}$ If pore chemistry is ignored for the sake of simplicity, the water viscosity and slip length increase for smaller-sized nanopores. Thus, the water flux across nanoporous graphene depends on the chemistry, size and geometry of the nanopores. ${ }^{12}$

Thomas et al. ${ }^{33}$ proposed that there are six main mechanisms for salt rejection by monolayer graphene membranes, including:

\section{1. size exclusion;}

2. dehydration effects (steric exclusion of the hydration shell);

3. charge repulsion;

4. subtler effects involving specific interactions with the pore, as observed in biological channels;

5. interactions of solutes with specific chemical structures of the pore; 6. entropic differences.

As the hydrated diameter of ions in water (for example, $\mathrm{Na}^{+}$ $0.72 \mathrm{~nm}, \mathrm{~K}^{+} 0.66 \mathrm{~nm}, \mathrm{Ca}^{2+} 0.82 \mathrm{~nm}, \mathrm{Mg}^{2+} 0.86 \mathrm{~nm}$ and $\mathrm{Cl}^{-}$ $0.66 \mathrm{~nm})$ is larger than the effective size of a water molecule 
$(0.26 \mathrm{~nm})$, the first and second mechanisms are the most important salt rejection mechanisms. ${ }^{6,12}$ Molecular dynamics simulations showed that monolayer graphene membranes with small enough nanopores can fully reject salt ions. ${ }^{30}$ Such studies determined that the critical nanopore diameter for rejecting $\mathrm{NaCl}$ lies between 0.6 and $0.8 \mathrm{~nm}$, in compliance with the hydration radius of the ions. ${ }^{34}$ In addition to size exclusion effect, the nanopore chemistry and thus the electrical charge of the nanopore edge also affect ion rejection. ${ }^{12}$ As shown by molecular dynamics simulations, ${ }^{35}$ the presence of negatively charged functional groups at the nanopore edge hampers the flux of negative ions $\left(\mathrm{Cl}^{-}\right)$, thereby enhancing the salt rejection efficiency. The fourth mechanism, that is, subtler pore/solute interactions, is directly correlated to the nanopore morphology and has been mainly studied regarding biological membranes rather than graphene membranes. Pore/solute effects, including overcoordination and undercoordination, can notably affect the ion selectivity of biological water channels. ${ }^{12}$ The fifth mechanism is based on the presence and interaction of chemical structures inside the nanopore with ions. In this regard, He et al., ${ }^{36}$ through molecular dynamics simulations, proved that a graphene nanopore with four carbonyl groups allows more permeation of $\mathrm{K}^{+}$than $\mathrm{Na}^{+}$, whereas a nanopore functionalized by four negatively charged carboxylate groups adsorbs $\mathrm{Na}^{+}$but allows the permeation of $\mathrm{K}^{+}$. These behaviors imply that collective effects between ions can impact the salt rejection of monolayer graphene. Moreover, the chemistry and shape of the nanopores are the determining factors of such behaviors. ${ }^{12}$ For instance, at moderate ionic strength, $\mathrm{NH}_{3}$-functionalized pores are unable to reject $\mathrm{Cl}^{-}$ions due to the accumulation of ions at the pore gate, whereas $\mathrm{OH}^{-}$-functionalized pores highly facilitate the removal of $\mathrm{Cl}^{-}$ions at both low and moderate ion concentrations, implying their optimum applicability for water desalination. ${ }^{15}$ Pores functionalized by negatively charged fluorine or nitrogen can ease the permeation of cations. ${ }^{37}$ On the other hand, nanopores terminated by positively charged hydrogen do the same for anions. ${ }^{15,37,38}$

The final mechanism is based on entropic differences induced by the graphene layer. Salt rejection is dependent on the free energy barrier through each nanopore. Accordingly, a monolayer graphene membrane can also desalinate water by limiting the number of physical arrangements (for example, geometric orientation) in which salt ions can permeate through the nanopores. For example, as mentioned earlier, hydrogenated nanopores demonstrate higher salt rejection than hydroxylated nanopores, because they decrease the number of possible geometric orientations and hydrogen-bonding configurations of hydrated ions. ${ }^{12}$

In practice, challenges and solutions. In addition to simulation-based efforts, numerous relevant experimental studies have also been performed. These studies proved the accuracy of simulation predictions regarding the high efficiency of graphene membranes for water desalination. Moreover, they addressed current challenges in the development of monolayer graphene membranes for industrial applications. In this regard, the main challenges include the following: (1) the low-cost production of large-area monolayer graphene; (2) the generation of high-density nanopores with controlled uniform sizes and chemical functionalities on the graphene sheet; and (3) the intrinsic and extrinsic defects formed during growth and graphene transfer processes.

Large-scale production. With respect to this challenge, large-area graphene has been grown on copper foils and the roll-to-roll transfer of 30-inch graphene has been realized. Bae et al. ${ }^{39}$ demonstrated graphene growth at atmospheric pressure via chemical vapor deposition (CVD) on large flexible copper foils. The synthesized graphene can be used in the form of a roll-type substrate. Such structure fits inside a tubular furnace to enhance the scale and homogeneity of the graphene films. Subsequently, due to the flexibility of graphene and the copper foil, efficient etching of the foil and the facile transfer of graphene comprise a cost- and time-effective roll-to-roll production method.

As shown in Figure 5, the main steps in this roll-to-roll transfer include the following:

(I) attaching a thin polymer support coated with an adhesive layer (for example, thermal release tape) to graphene on the copper foil by passing between two rollers;

(II) etching of the copper foil by a $0.1 \mathrm{M}$ ammonium persulphate aqueous solution $\left(\left(\mathrm{NH}_{4}\right)_{2} \mathrm{~S}_{2} \mathrm{O}_{8}\right)$;

(III) detachment of the graphene layer and transfer onto a substrate. This step is performed by removing the adhesive force holding the graphene film, for example, via thermal treatment.

Bae et al. ${ }^{39}$ stated that, due to the scalability and processability of CVD graphene and their roll-to-roll method, the continuous production of large-scale graphene-based devices is feasible.

Formation of nanopores in a controlled manner. Pores in monolayer graphene structures can be intrinsic or synthesized through different approaches. The formation of intrinsic pores in graphene could result from defects in the copper foil catalyst substrate or the deposition and growth of particulates on the copper substrate.

Regarding employing monolayer graphene membranes with intrinsic pores, O'Hern et al. ${ }^{28}$ transferred a monolayer of CVD graphene onto a porous polycarbonate substrate to fabricate a graphene composite membrane as large as $25 \mathrm{~mm}^{2}$. The intrinsic $1-15 \mathrm{~nm}$

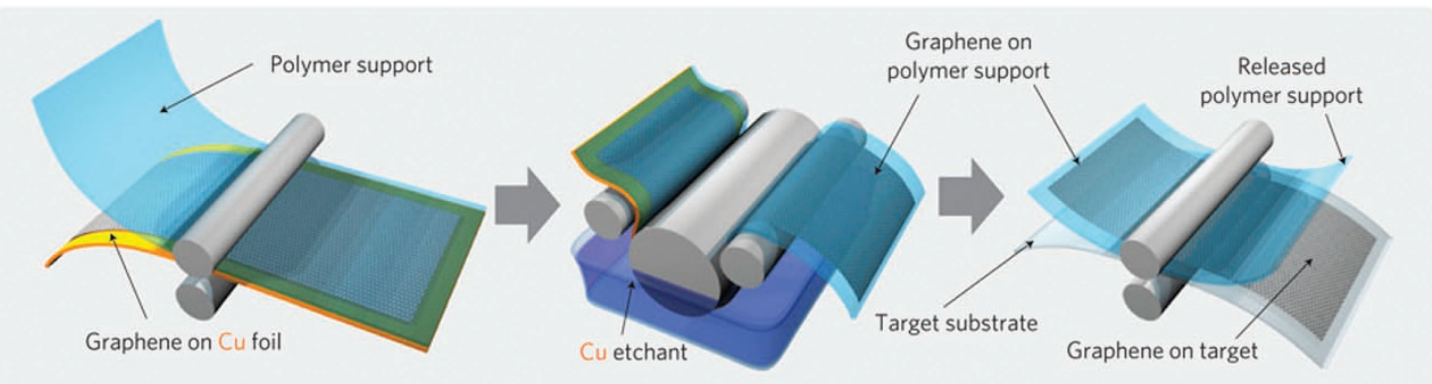

Figure 5 Schematic of the roll-to-roll transfer process illustrating the steps of adhesion of the polymer support, copper etching (rinsing) and dry transferprinting on a target substrate. (Reprinted with permission from Bae et al. ${ }^{39}$ Copyright 2016 Nature Publishing Group.) 
diameter pores in the CVD graphene contributed to the size-selective transport of molecules through the membrane. The pore size distribution, obtained by scanning transmission electron microscopy, reveals that $\sim 83 \%$ of the pores are smaller than $10 \mathrm{~nm}$ in diameter. The authors experimentally measured the diffusive transport of molecules with different sizes, including $\mathrm{KCl}$, tetramethylammonium chloride, Allura Red AC (496 Da dye) and tetramethylrhodamine dextran $(70 \mathrm{kDa})$, across the membrane. The graphene composite membrane allowed the permeation of $\mathrm{KCl}$ and tetramethylammonium chloride but blocked the diffusion of tetramethylrhodamine dextran $(12 \mathrm{~nm})$. The diffusive transport of the larger tetramethylrhodamine dextran molecules was one order of magnitude lower than that of the smaller molecules. The Stokes-Einstein molecular diameters of Allura Red and tetramethylrhodamine dextran are $\sim 1$ and $\sim 12 \mathrm{~nm}$, respectively, implying that the pores are mostly $1-10 \mathrm{~nm}$ in size, as confirmed by the scanning transmission electron microscopy observations. The measured permeability agreed with that obtained via continuum model predictions for graphene with a porosity of $0.012-0.61 \%$ and intrinsic pore size of $1-10 \mathrm{~nm}$, that is, $0.025-$ $0.15 \%$. The authors claimed that selective molecular transport through macroscopic areas of the monolayers of CVD graphene is completely feasible. However, this selectivity cannot be controlled due to random sizes and locations of the intrinsic pores. Thus, the generation of highdensity nanopores with controlled uniform sizes and chemical functionalities on graphene layers is a challenging task that must be addressed in the development of graphene membranes. In this regard, Surwade et al. ${ }^{40}$ synthesized a monolayer graphene membrane through ambient pressure CVD on a copper foil catalyst. In this membrane, nanopores were precisely incorporated in a suspended monolayer graphene by an oxygen plasma etching process, enabling adjustment of the pore size. Nanopores generated in such a way enhanced the water molecule selectivity of the membrane over dissolved ions, including $\mathrm{K}^{+}, \mathrm{Na}^{+}, \mathrm{Li}^{+}$and $\mathrm{Cl}^{-}$. The membrane, plasma etched for a short exposure time, was successful in the rejection of salt (100\%) with high water permeability. Although the selectivity exceeded five orders of magnitude at low porosities (proportional to the plasma treatment duration), the selectivity declined at higher porosities, most likely due to growth of the nanopores. When a pressure difference was the driving force, a water flux of $10^{6} \mathrm{~g} \mathrm{~m}^{-2} \mathrm{~s}^{-1}$ at $40^{\circ} \mathrm{C}$ was obtained. Considering the nanopore density of $\sim 1 / 100 \mathrm{~nm}^{2}$, the water flux through a single nanopore can be as high as three molecules per picosecond. This flux is an order of magnitude higher than that calculated by molecular dynamics simulations of the same pore size.

In addition to the oxygen plasma etching process, graphene pore sizes as small as those determined via simulations were recently created by ion and electron bombardment. ${ }^{41}$ Single, controlled pores smaller than $2 \mathrm{~nm}$ were fabricated by irradiating graphene with a focused electron beam above the carbon knockout potential $(80 \mathrm{kV}) .{ }^{42}$ Pores in larger areas of graphene can also be created by oxidative processes, ${ }^{43-45}$ for example, via exposure to high-temperature atmospheric oxygen ${ }^{46}$ ozone under UV light ${ }^{43,44}$ and hydrogen plasma. ${ }^{45}$ However, considering the higher reactivity of grain boundaries compared with the basal plane, the pores created by oxidation processes have very discrepant sizes. ${ }^{45}$ To create pores of controlled size and density, the reactivity difference of the basal plane and the grain boundaries can be alleviated by the inclusion of artificial defects in the basal plane via argon ion irradiation. ${ }^{41}$ To enlarge the defects in the pores, defective graphene is irradiated with an electron beam at the carbon knockout potential. This technique is more efficient in the creation of tightly distributed pore sizes than oxidative processes. In such conditions, however, pores are formed over a limited membrane area due to the application of a high-voltage electron beam for pore enlargement. ${ }^{47}$ In this regard, O'Hern et al. ${ }^{47}$ reported selective ionic transport through controlled, high-density, subnanometer pores in a macroscopic monolayer CVD graphene membrane. First, isolated reactive defects were incorporated into the graphene lattice by gallium ion bombardment at a density of $\sim 6 \times 10^{12}$ ions per $\mathrm{cm}^{2}$. Subsequently, the defects were enlarged by oxidative etching (in acidic i. Graphene on TEM grid

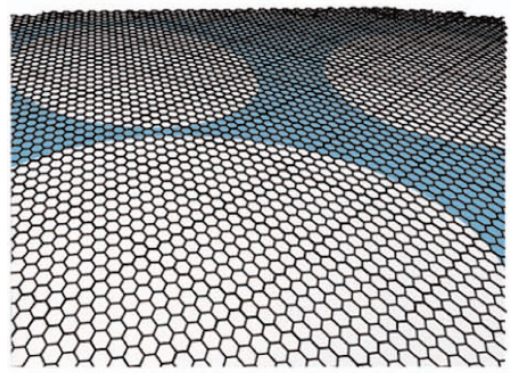

iii. Bombardment creates defects

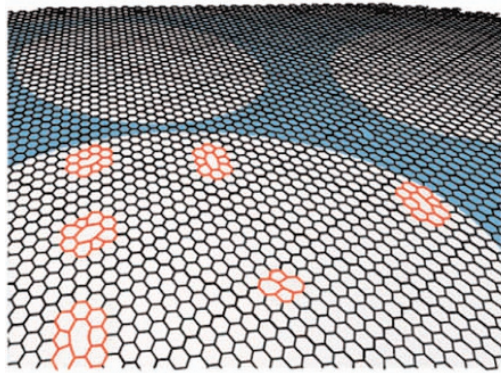

\section{ii. Bombard with Gallium lons}

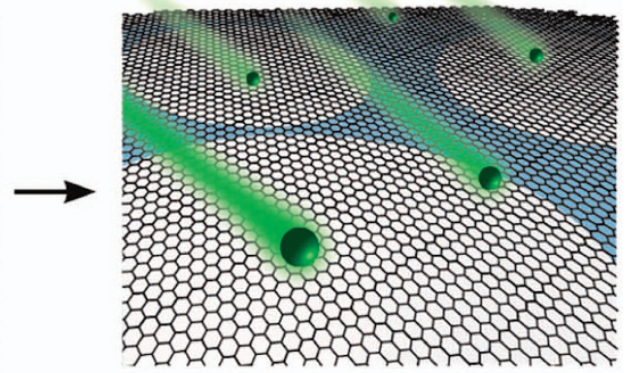

iv. Defects grow into holes

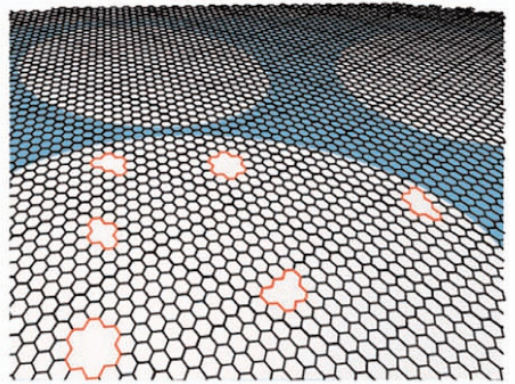

Figure 6 Schematic illustrating the process for the inclusion of controlled pores in a monolayer graphene membrane by ion bombardment followed by chemical oxidation. (Reprinted with permission from O'Hern et al. ${ }^{47}$ Copyright 2016 American Chemical Society.) 
potassium permanganate) into permeable pores as large as $0.40 \pm 0.24 \mathrm{~nm}$ with a density over $10^{12} \mathrm{~cm}^{-2}$ (Figure 6). Through diffusion measurements of $\mathrm{KCl}$ and an organic dye, control over the selectivity of the monolayer graphene membrane at the subnanometer scale was proven. According to transport measurements on the ionirradiated graphene membranes, the in situ etched pores formed at short oxidation times were cation-selective. This behavior is attributed to the electrostatic repulsion of anions by the negatively charged functional groups present at the pore edges, such as ketone, quinone, hydroxyl or carboxyl groups. At longer oxidation times and thus further growth of the pores, electrostatic effects determining the $\mathrm{K} / \mathrm{Cl}$ ion selectivity diminished. Accordingly, the pores enabled the transport of salt but hampered that of larger organic molecules, indicating the steric size exclusion ability of the graphene membrane. In general, as shown in Figure 7a, size exclusion and electrostatic interactions are the main separation mechanisms in monolayer graphene membranes.

Sealing the intrinsic and extrinsic defects. Ideally, a monolayer graphene membrane should possess only homogenously sized pores at high density. However, intrinsic and extrinsic defects formed during growth and graphene transfer processes, ${ }^{28,47,48}$ respectively, act as leakage pathways and hinder the practical realization of graphene monolayer membranes. Accordingly, such defects must be sealed or blocked or molecular permeation through them should be hampered. O'Hern et al. ${ }^{49}$ suggested an advanced approach to seal defects while maintaining a large part of a centimeter-scale monolayer graphene membrane usable for the NF of salts and small molecules. Mass transport measurements and electron microscopy indicated that this membrane includes nanometer-scale $(\sim 1-15 \mathrm{~nm})$ intrinsic defects that formed during CVD on copper and large $(\sim 100-200 \mathrm{~nm})$ tears that formed during graphene transfer. ${ }^{28}$ The intrinsic defects were sealed by selectively filling with hafnia via atomic layer deposition (Figure 8). In addition, an interfacial polymerization process was adopted to seal large defects via deposition of nylon-6,6 within the defects. This leakage-sealing process was evaluated by the diffusion of $\mathrm{KCl}$ across the membrane. ${ }^{28}$ Although monolayer graphene mounted to a polycarbonate track etch membrane decreases the permeation of $\mathrm{KCl}$ to $\sim 65 \%$ of that across a bare polycarbonate track etch

a Nanoporous graphene membrane

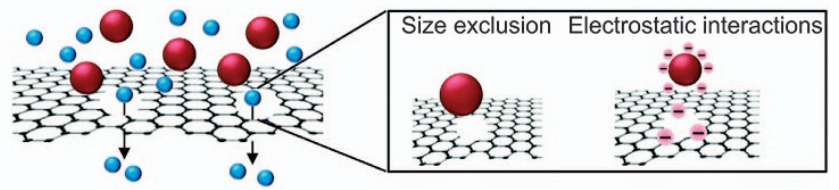

b

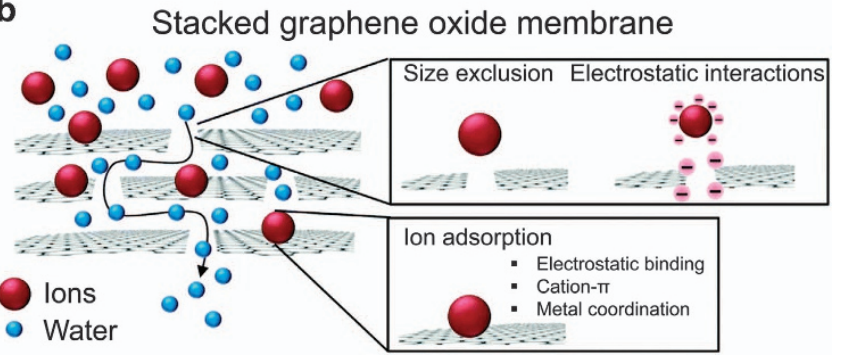

Figure 7 Schematic of the separation mechanism of (a) a monolayer graphene membrane with nanopores of controlled size and (b) a multilayer graphene membrane composed of stacked GO sheets. (Reprinted with permission from Perreault et al. ${ }^{24}$ Copyright 2016 The Royal Society of Chemistry.) membrane, defect-free monolayer graphene can decrease this flux to zero. In this regard, the deposition of hafnia on graphene lowers the flux to $\sim 40 \%$ and subsequent interfacial polymerization further decreases it to $\sim 8 \%$ of that through a bare polycarbonate track etch membrane, showing a significant sealing ability for the graphene layer. Subsequently, pores were introduced into the graphene by high-energy gallium ion bombardment, nucleating isolated defects, and then etching the defects using acidic potassium permanganate ${ }^{47}$ According to aberration-corrected scanning transmission electron microscopy, distinct pores as small as $0.162 \mathrm{~nm}$ with a density of $3.89 \times 10^{13} \mathrm{~cm}^{-2}$ were formed. Recalling that the van der Waals diameter of a water molecule is $0.275 \mathrm{~nm}$, only the larger pores with a density of $1.57 \times 10^{12} \mathrm{~cm}^{-2}$ can permeate water and almost all of the pores should block salt ions $(\sim 0.7 \mathrm{~nm} \text { in diameter) })^{30}$ The separation ability of the graphene membrane was examined using a forward osmosis process with different solutes, such as $\mathrm{NaCl}(0.716 \mathrm{~nm}$ size $), \mathrm{MgSO}_{4}$ (0.86 nm size), Allura Red (496 Da and $\sim 1.0 \mathrm{~nm}$ size) and dextran ( $4.4 \mathrm{kDa}$ and $\sim 3.7 \mathrm{~nm}$ size). The rejection efficiency of the membrane was as follows: $\sim 70 \%$ of $\mathrm{MgSO}_{4}, \sim 90 \%$ of Allura Red and $\sim 83 \%$ of dextran. Surprisingly, $\mathrm{NaCl}$ showed a negative rejection, implying that the permeation rate of $\mathrm{NaCl}$ through the membrane exceeds that for non-selective convective flow. This behavior may be correlated to the presence of few-nanometer-scale defects, ${ }^{28}$ which are too large to be sealed using atomic layer deposition and too small to allow blocking by interfacial polymerization, as well as to the enhanced permeability of nylon-6,6 to monovalent ions during the etching step of pore creation.

\section{Multilayer graphene desalination membranes}

Despite the significant advantages of monolayer graphene membranes, especially in terms of water permeability, the fabrication of leak-free, large-area monolayer graphene membranes with controlled pore density and size on the industrial scale is challenging. ${ }^{47,49,50}$ One solution for this challenge is the fabrication of desalination membranes based on stacked GO nanosheets. These nanosheets are highly stackable, mainly due to their structure, a single-atom-thick layer with a lateral dimension reaching tens of micrometers. Durable interlayer hydrogen bonds hold the GO sheets together to form a stable freestanding membrane. ${ }^{51}$ Moreover, GO nanosheets can be produced on a large scale with low cost via chemical oxidation and the ultrasonic exfoliation of graphite. This method promises the costefficient and industrially applicable fabrication of stacked membranes. Finally, 2D graphene offers not only extraordinary chemical and thermal stabilities but also superior flexibility and solution processibility. ${ }^{21,52}$

As shown in Figure 7b, GO sheets can be arranged as highly ordered films with 2D nanochannels between two adjacent graphene sheets. ${ }^{51,52}$ In this structure, the $2 \mathrm{D}$ channels enable the permeation of water while rejecting undesired solutes. In addition, the presence of oxygen-containing functional groups, such as carboxyl groups, on the GO nanosheets enables functionalization and thus enables related charge-based interactions with water pollutants. ${ }^{18}$ Such promising features make multilayer GO structure an ideal candidate for the production of advanced ionic and molecular sieving membranes for desalination. ${ }^{1,4,53-56}$

Theoretical considerations. According to molecular simulations, water flows at a very high rate in planar graphene nanochannels because of the very large slip length and low friction on graphene sheets. ${ }^{28,57}$ The rate of water flow through the frictionless, hydrophobic, pristine (non-oxidized) region of the channels has been estimated to be as high 
a

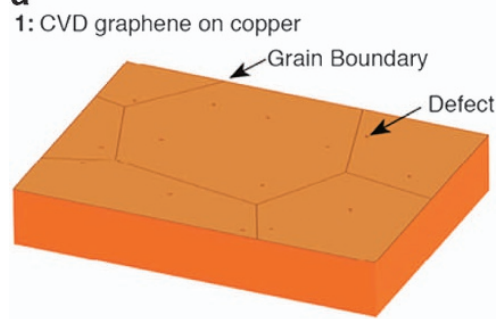

3: Atomic layer deposition of $\mathrm{HfO}_{2}$

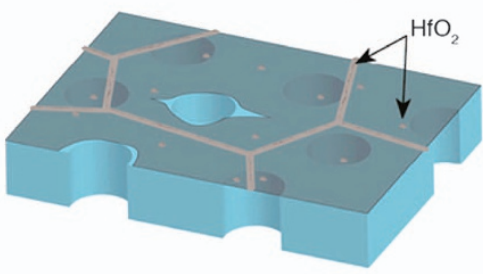

2: Transfer to PCTE membrane

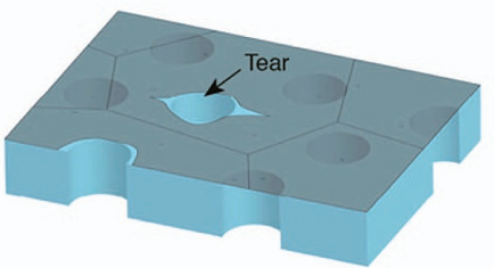

4: Interfacial polymerization (IP) of nylon 6,6

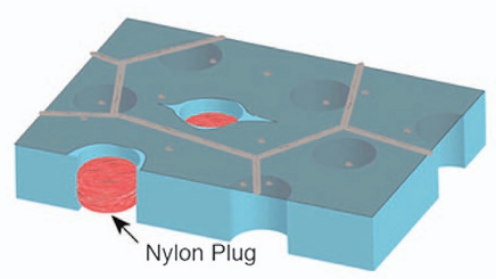

b
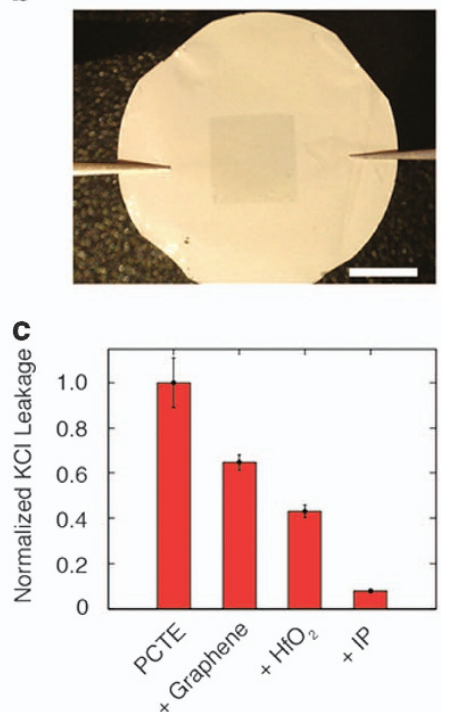

Figure 8 Schematic of the defect sealing process for a monolayer graphene membrane. (a) (1) Graphene, containing grain boundaries and pinhole defects, is formed by CVD on a copper substrate and then (2) transferred to a polycarbonate track etch (PCTE) membrane via pressing the graphene-on-copper onto the PCTE membrane and removing the copper via etching. (3) The nanoscale defects and leaks are sealed by the deposition of $\sim 3.5 \mathrm{~nm}$ of hafnia onto the graphene surface using atomic layer deposition (ALD). (4) Furthermore, through an interfacial polymerization process, nylon-6,6 is formed in the PCTE pores underlying larger defects. (b) Optical image of the final membrane, wherein the graphene layer is clearly seen at the center of the PCTE membrane. The scale bar is $0.5 \mathrm{~cm}$. (c) The diffusive permeation of $\mathrm{KCl}$ through the control membrane (no pores created) normalized by the permeation rate through a bare PCTE membrane decreases with each successive sealing step. (Reprinted with permission from O'Hern et al. ${ }^{49}$ Copyright 2016 American Chemical Society.)

as $20 \mathrm{~m} \mathrm{~s}^{-1.56}$ This high water permeability is also attributed to capillary-driven forces in the $2 \mathrm{D}$ channels of pristine graphene sheets. ${ }^{58}$ In contrast, due to hydrogen-bonding interactions between water molecules with oxygen-containing functional groups, the fast water flow slows between the hydrophilic, oxidized graphene sheets. ${ }^{58}$ In addition to water, ions smaller than the size of the nanochannels can permeate through the GO membrane much faster than via simple diffusion. This fast permeation is due to the high capillary pressure imposed on the ions inside the graphene channels. In addition to the high rate of water flow through the nanochannels, water permeates quickly through the entire GO membrane as well. This behavior is attributed to the porous microstructure of the membrane, including the open space between the edges of adjacent GO sheets, the wide channels formed at wrinkles and the presence of holes on the GO sheets. ${ }^{18}$ In the latter case, as mentioned earlier, the average areal percentage of holes in a GO sheet is only $2 \%$. Therefore, the holes will very likely be blocked by the impermeable, oxidized and pristine regions of the adjacent GO sheets. For instance, a layered GO membrane with a thickness not exceeding $1 \mu \mathrm{m}$ should contain over one thousand GO sheets; as a result, holes do not notably contribute to the permeability of the membrane. ${ }^{59}$ Notably, the permeability also strongly depends on the thickness of the membrane. Huang et al. ${ }^{60}$ stated that a thicker graphene membrane can lead to a lower permeability, mainly due to its longer channel tortuosity.

Size exclusion is assumed to be the main and dominant separation mechanism. ${ }^{55}$ In this regard, the interlayer spacing of the GO sheets has a key role. Although in graphite, which is composed of pristine graphene sheets, the interlayer spacing in dry state is only $3.4 \AA$, the presence of oxygen-containing functional groups on the GO sheets increases this spacing to $6-7 \AA .^{18,61}$ As the humidity increases, water molecules penetrate into the interlayer space between the GO nanosheets, thus expanding the spacing. In the wet state, when GO laminates are soaked in water, the interlayer separation further increases, leading to the passage of two or three water layers. As Wei et al. ${ }^{58}$ reported, there is a correlation between the formation of mono-, bi- and trilayer water and an interlayer distance below 0.7, 1.0 and $1.4 \mathrm{~nm}$, respectively.

The entrance of the interlayer space of the GO membrane for ions and molecules is the edge of the GO sheets. As oxygen-containing functional groups are prone to clustering, they induce puckering and thus corrugation of the sheets. ${ }^{62}$ This behavior gives rise to the formation of large, percolating regions of non-oxidized graphene. ${ }^{56}$ Therefore, the GO sheets will contain empty spaces located between non-oxidized regions of the graphene sheets, ${ }^{56}$ forming a network of pristine graphene channels inside the GO sheets. This fact indicates that the interlayer spacing of the GO sheets and thus the diffusivity of ions and molecules depend on the presence of oxidized and nonoxidized domains. The largest interlayer space is seen between the GO sheets at the facing pristine domains. Thus, the diffusion rate of ions and molecules is enhanced. Meanwhile, the interlayer spacing between the facing pristine and oxidized domains is decreased. This smaller interlayer space, along with the possibility of hydrogen bonding and electrostatic interactions in the oxidized regions, results in the lower mobility of molecules and ions. ${ }^{58}$ The interlayer spacing between the GO sheets with oxidized facing domains is the narrowest, thus blocking molecules and ions. ${ }^{56,63}$

Ions and molecules inside the network of interlayer nanochannels diffuse parallel to the GO sheets. However, at the gap between the edges of non-interlocked neighboring GO sheets and, at the cracks and holes of the sheet, they can diffuse perpendicular to the GO sheets and permeate through the membrane. ${ }^{18,55}$ Thus, such structural defects supplement the role of the interlayer nanochannels in the separation of the ions and molecules. Moreover, oxygen-containing functional groups on the GO sheets are ionized in the wet state and thus negatively charged. This fact influences the anion and cation mobility. ${ }^{18}$ Thus, the selectivity of the GO multilayer membranes 
results from a combination of size-exclusion effects depending on the interlayer spacing of the GO sheets, electrostatic interactions between the ions and negatively charged GO sheets, and ion adsorption effects, such as cation- $\pi$ interactions and metal coordination to the GO sheets, as shown in Fig. $7 \mathrm{~b} .^{24}$

In addition to the size-exclusion mechanism, ions can be separated from water by stacked graphene membranes through electrostatic interactions with the GO sheets. In a related study, ${ }^{64}$ the diffusion properties of various ions in different sodium salt solutions through a GO membrane were determined. When applying a $\mathrm{NaOH}$ solution, $\mathrm{OH}^{-}$interacts with the carboxyl and hydroxyl groups on the GO sheets, making them highly ionized. Accordingly, electrostatic repulsion forces between the GO sheets increase, leading to an increase in the interlayer spacing. This occurrence leads to the fast permeation of $\mathrm{OH}^{-}$and $\mathrm{Na}^{+}$within the membrane. In the case of $\mathrm{NaHSO}_{4}$ solution, the $\mathrm{H}^{+}$cations inhibit the ionization of carboxyl and hydroxyl groups, and thus the interlayer spacing remains small, slowing the permeation of ions. Transition-metal cations can also be adsorbed onto the GO sheets through coordination bonds to $s p^{3}$ clusters in the oxidized regions of the GO sheets, whereas alkali metal cations can be adsorbed through cation- $\pi$ interactions with $s p^{2}$ clusters in the pristine regions of the GO sheets. ${ }^{65}$

The sieving and electrostatic-based separation performance of the multilayer graphene membranes can be optimized by several modifications. For instance, the GO sheet size and membrane thickness can be changed to tune the separation efficiency of the membrane. When utilizing nanosized GO sheets, a larger number of gaps between the edges of non-interlocked neighboring GO sheets is created compared to microsized GO sheets. Accordingly, the permeation rate of molecules or ions through the GO membrane perpendicular to the GO sheets is notably faster. ${ }^{66}$ In thin membranes, large pores ( $>1.75 \mathrm{~nm}$ in diameter) created by vacancies, edges and cracks within the single GO sheets facilitate ion permeation through the membranes. In thick membranes, the interlayer spacing between the GO sheets is the determining factor for ion penetration. ${ }^{55,67}$ Thus, by changing the number of stacked GO sheets in the fabrication step, the separation efficiency of ions and molecules can be adjusted. Moreover, the $\mathrm{pH}$ of water can affect the ionization level of the carboxyl and hydroxyl groups on the GO sheets. When ionized, the GO sheets become negatively charged and repel each other. This behavior leads to the expansion of the interlayer space and indirectly adjusts the separation efficiency of the GO membrane. ${ }^{68}$ A hybridization strategy can also provide better permeation performance of the GO stacked membrane. By the inclusion of positively charged $\mathrm{Cu}(\mathrm{OH})_{2}$ nanostrands or nanosized carbon dots in a GO stacked membrane, the water permeability rises several-fold higher. In addition, such an approach does not compromise the rejection efficiency of the membrane for pollutants such as organic dyes. ${ }^{69,70}$

In practice, challenges and solutions. Although numerous studies have shown the applicability of this class of membranes for water desalination, in particular as NF membranes, many studies in the literature have addressed current challenges. There are several challenges ahead of the industrial application of multilayer graphene membranes. These challenges include the following: (1) swelling, (2) water instability, (3) low mechanical durability and (4) scalability. Moreover, such membranes have shown solely a limited NF desalination capability. Thus, to extend the applicability from NF desalination to seawater desalination, ion rejection must be further optimized.

Hydration-induced swelling of the GO nanosheet spacing. In the fully hydrated state, two or three water layers are present inside the channels of the GO sheets, such that $\mathrm{Na}^{+}$and $\mathrm{Cl}^{-}$ions can permeate through the membrane to achieve desalination (Figure 9). Nair et al. ${ }^{56}$ reported that dry-state GO membranes synthesized via a vacuum filtration process possess an extremely packed structure, and the void spacing between GO layers cannot exceed $0.3 \mathrm{~nm}$. In such a compact structure, only water vapor aligned in a monolayer can permeate through the nanochannels. However, when this structure is immersed in an ionic solution, due to hydration, the GO spacing can be enlarged to $0.9 \mathrm{~nm} .{ }^{55}$ Accordingly, any species, including ions or molecules, possessing a hydrated radius of $0.45 \mathrm{~nm}$ or less can penetrate into the nanochannel, whereas larger species are readily hampered (Figure 10a). Conclusively, the swelling of GO laminates in water has an adverse effect on the rejection of ions and must be prevented for desalination.

For desalination, the GO spacing should not exceed $0.7 \mathrm{~nm}$ to filter out hydrated $\mathrm{Na}^{+}$, which has a hydrated radius of $0.36 \mathrm{~nm}$. Thus, the spacing must be accordingly tuned, and the problem of swelling must be overcome. Relevant strategies include the partial reduction of GO to shrink the size of the hydrated functional groups and covalent bonding of the GO nanosheets by tiny molecules to resist the hydration force (Figure 10b). On the other hand, for water purification, wastewater reuse and pharmaceutical and fuel separation, the GO spacing can be increased to as large as $1-2 \mathrm{~nm}$ by the inclusion of large, rigid chemical groups ${ }^{31}$ or soft polymer chains, for example, polyelectrolytes between the GO nanosheets. If an even larger spacing

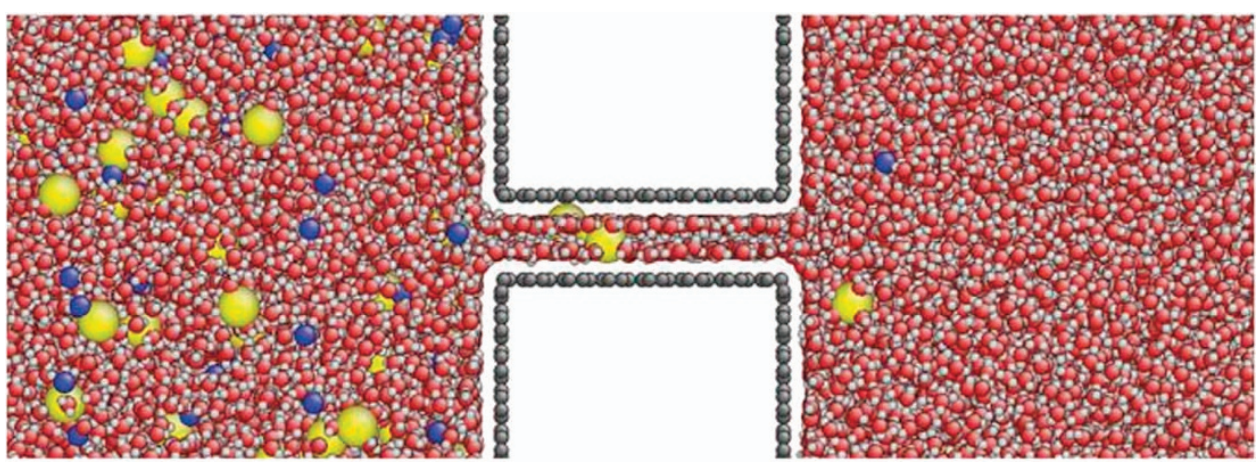

Figure 9 Simulation of molecular sieving by a GO multilayer membrane. $\mathrm{NaCl}$ diffuses through a $9 \AA$ graphene slit encompassing two layers of water. The $\mathrm{Na}^{+}$and $\mathrm{Cl}^{-}$ions are shown in yellow and blue, respectively. (Reprinted with permission from Joshi et al. ${ }^{55}$ Copyright 2016 The American Association for the Advancement of Science.) 
a Transport of ions and molecules in the GO membrane

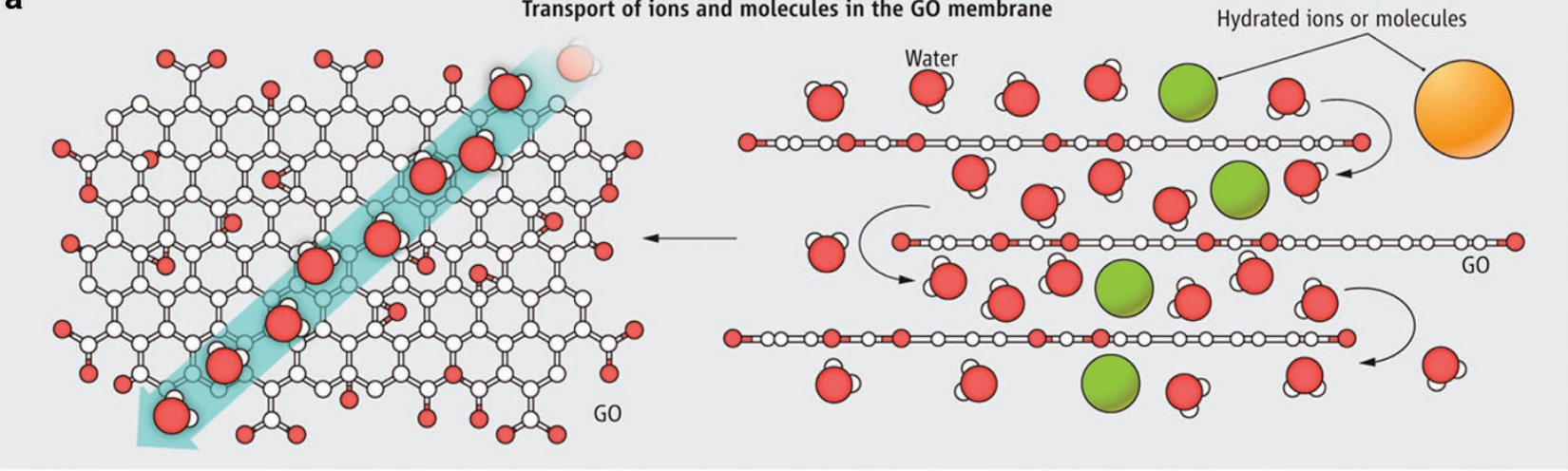

b Desalination or hydrofracking

Covalent bonds or small functional groups

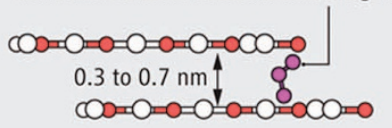

Water, fuel, or chemical purification

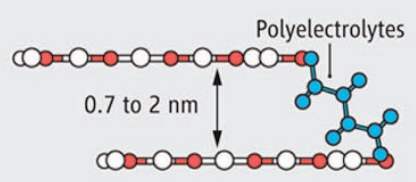

Biomedical filtration

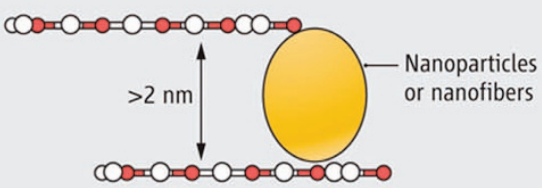

C

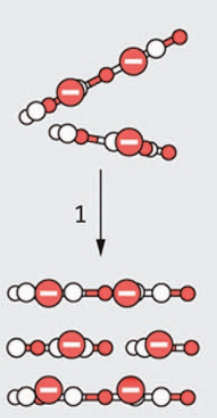

Vacuum filtration

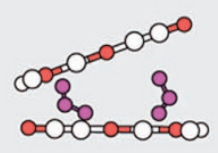

2

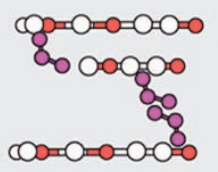

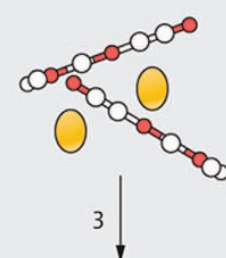

$\mathrm{COO}=\mathrm{O}=\mathrm{O}=\mathrm{O}=\mathrm{O}=0$

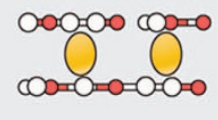

Layer-by-layer assembly
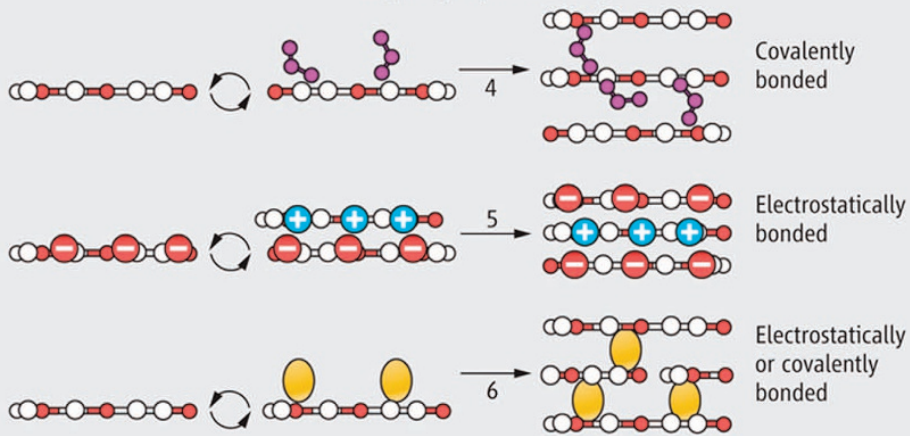

Figure 10 (a) Species smaller than the spacing between GO nanosheets, including water, ions and molecules, quickly permeate through the GO membrane, while larger species are hindered. (b) The nanochannel size and thus separation efficiency of the GO membrane can be adjusted. (c) To fabricate GO membranes, GO nanosheets can be either physically packed by vacuum filtration (options 1 to 3), or stabilized by covalent bonds, electrostatic forces, or both (options 4 to 6) during layer-by-layer (LbL) assembly. (Reprinted with permission from Mi. ${ }^{53}$ Copyright 2016 The American Association for the Advancement of Science.)

is required, for example, in biomedicine for artificial kidneys and dialysis, wherein large biomolecules and small waste molecules are separated, nanoparticles and nanofibers can be incorporated in the membrane structure. To tune the GO nanosheet spacing and thus the separation performance of the membrane, Burress et al. ${ }^{71}$ proposed an efficient strategy. They developed a graphene membrane based on GO sheets covalently interconnected by linkers, such as linear boronic acid pillaring units. Such a hybrid material incorporates not only the hydrophobicity of graphene and thus the high water permeability but also the superior filtration efficiency induced by a tunable linker concentration. According to molecular dynamics simulations, this GO framework membrane (10 nm in thickness) could perfectly reject salt and its water permeability was two orders of magnitude higher than that of existing RO systems. Using a similar thickness to conventional RO membranes, that is, $100 \mathrm{~nm}$, a 10 -fold higher performance was achieved by the GO framework membrane. In addition, depending on the size of the particles to be rejected, that is, from micro-filtration to $\mathrm{NF}$, the water permeability and molecule rejection of the membrane could be tuned by adjusting the linker concentration and membrane thickness.

Water instability of multilayer graphene membranes. The presence of hydrated functional groups degrades the stability of $\mathrm{GO}$ in water. The oxidized regions act as spacers to separate adjacent GO sheets and allow water molecules to intercalate between the GO sheets. ${ }^{72,73}$ The instability of GO structures in water is the main challenge ahead of their application in aqueous media as separation membranes, as GO structures disintegrate over time. ${ }^{74}$ For application to water treatment, GO membranes should be stabilized by reduction or chemical crosslinking. ${ }^{72}$ The abovementioned crosslinking not only alleviates the swelling problem of GO membranes but also stabilizes the membranes. Moreover, by enhancing the $\pi-\pi$ interactions between GO sheets via a reduction process, the stability of the GO membranes is improved. Reduction of GO membranes by strong reductants, such as hydrazine, vitamin $\mathrm{C}$ and so on or by hydrothermal treatment can remove oxygen-containing functional groups. ${ }^{72,75}$ This process 
therefore shrinks the interlayer space between GO sheets and increases the barrier properties of the membrane.

In addition to chemical modification of the GO sheets, the assembly process can be a determining factor for the stabilization of the membrane's structure. As shown in Figure $10 \mathrm{c}$, vacuum filtration and layer-by-layer assembly are conventional synthetic methods for layered GO membranes. These eco-friendly methods are performed in aqueous solution without the need for organic solvents. Due to the high hydrophilicity of GO, GO membranes prepared via vacuum filtration of a pure GO solution or a mixture of GO and spacers suffer from weak bonding between the GO nanosheets. Thus, the nanosheets are dispersed in water during conventional cross flow filtration. In contrast, due to the presence of strong interlayer forces supplied by covalent bonding ${ }^{31}$ and/or electrostatic interactions during layer deposition, the layer-by-layer method is regarded as a superior approach. Moreover, this method is optimally scalable and cost effective. As an example, using multilayer graphene membranes produced via layer-by-layer assembly, $\mathrm{Hu}$ and $\mathrm{Mi}^{31}$ developed a new kind of NF membrane using GO nanosheets. The membrane was constructed via the layer-by-layer deposition of GO nanosheets on a polydopamine-coated polysulfone support. Owing to the presence of a large number of oxygen-containing groups on GO that can react with the amine groups of polydopamine, the GO nanosheets are strongly adhered on the support surface, thus forming a more robust and stable GO membrane. The GO nanosheets were previously cross-linked by 1,3,5-benzenetricarbonyl trichloride not only to overcome their dispensability in water (due to their high hydrophilicity) but also to
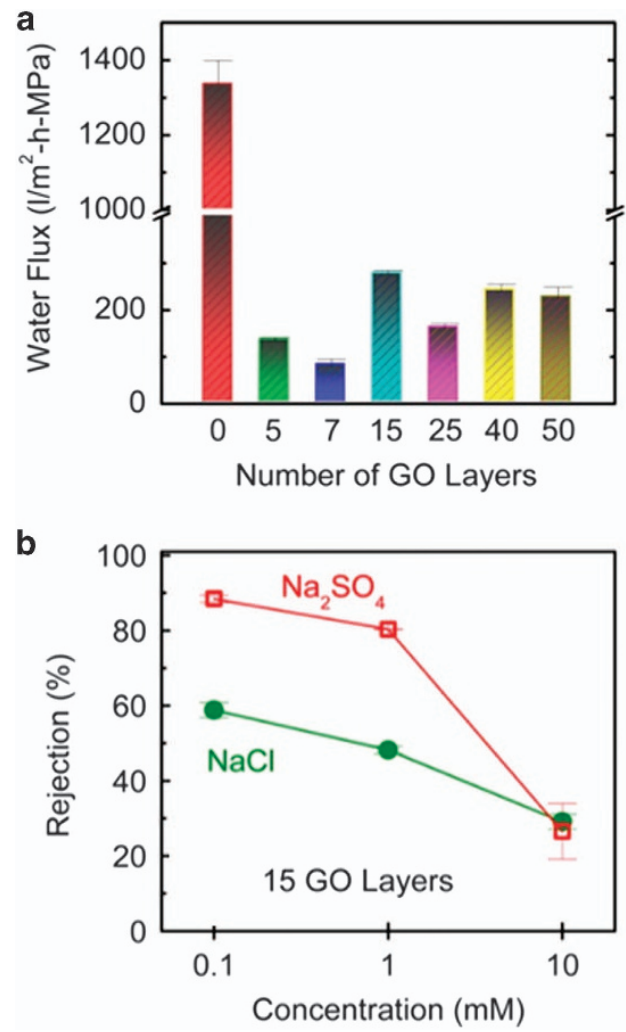

Figure 11 Water filtration performance of a GO membrane: (a) water flux versus the number of GO layers and (b) effect of the salt concentration on rejection by a 15 -layered GO membrane. It is noteworthy that 0 layer represents the polydopamine-coated membrane. (Reprinted with permission from $\mathrm{Hu}$ and Mi. ${ }^{31}$ Copyright (2016) American Chemical Society.) adjust their charges, functionality and spacing. As seen in Figure 11a, under a pressure of $50 \mathrm{psi}$, the GO membrane showed a water permeability of $80-2761 \cdot \mathrm{m}^{-2} \cdot \mathrm{h}^{-1} \cdot \mathrm{MPa}^{-1}$, which is $4-10$ times higher than that of most commercial NF membranes. Interestingly, the water flux does not decrease monotonically with the number of GO layers, implying no direct correlation between the membrane thickness and the water flow resistance. The reason for this could be the extraordinary water transport properties of the GO nanochannels. Regarding the water desalination performance of the membrane, the authors employed $\mathrm{NaCl}$ and $\mathrm{Na}_{2} \mathrm{SO}_{4}$ as representative mono- and divalent salts, respectively. The rejection efficiency of the GO membrane for $\mathrm{NaCl}$ and $\mathrm{Na}_{2} \mathrm{SO}_{4}$ was $6-19 \%$ and $26-46 \%$, respectively.

$\mathrm{Hu}$ and $\mathrm{Mi}^{31}$ investigated the influence of electrostatic charges on the separation efficiency of GO membranes by considering different $\mathrm{NaCl}$ and $\mathrm{Na}_{2} \mathrm{SO}_{4}$ solution concentrations. As seen in Figure $11 \mathrm{~b}$, the higher the ionic strength (solution concentration), the lower the rejection efficiency. When the $\mathrm{Na}_{2} \mathrm{SO}_{4}$ concentration is increased from $0.1 \mathrm{~mm}$ to $10 \mathrm{~mm}$, its rejection efficiency decreases from 88 to $26 \%$. For the same concentration limits, the $\mathrm{NaCl}$ rejection efficiency decreases from 59 to $29 \%$. This implies the significant influence of the charge effects on the separation efficiency of the GO membrane. To explain this behavior, the Debye lengths of the salt solutions should be considered. The Debye lengths of $0.1 \mathrm{~mm} \mathrm{NaCl}, 0.1 \mathrm{~mm} \mathrm{Na}_{2} \mathrm{SO}_{4}$, $10 \mathrm{~mm} \mathrm{NaCl}$ and $10 \mathrm{~mm} \mathrm{Na}_{2} \mathrm{SO}_{4}$ are 31, 18, 3.1 and $1.8 \mathrm{~nm}$, respectively. When the Debye length decreases, the electrostatic repulsion between the ions and the charged membrane decreases due to the thinning of the electrostatic double layers. Accordingly, the related rejection efficiency decreases.

To stabilize as well as minimize the hydration-induced swelling of GO channels, very recently, Abraham et al. ${ }^{76}$ suggested an innovative physical approach. They prevented swelling by exposing the GO membrane strips to a given humidity to attain a certain interlayer spacing and subsequently embedding the GO laminate in epoxy. Using this gluing approach, they succeeded in preventing further dimensional changes in the GO membrane when hydrated. The epoxy embedment did not alter the spacing, which remained fixed even when the epoxy was removed. Permeation experiments using $\mathrm{K}^{+}, \mathrm{Na}^{+}$, $\mathrm{Li}^{+}, \mathrm{Ca}^{2+}$ and $\mathrm{Mg}^{2+}$ cations over an extensive temperature range showed an exponential decrease in the ion permeation rate with decreased spacing. Interestingly, only a linear decrease in the water permeation was observed. This behavior can be explained by considering that an ion traveling through a GO membrane carries some baggage, that is, water molecules stuck to the ion as a hydration shell. Thus, to pass through the capillaries, the ion must lose some of its excess baggage and overcome the dehydration energy barrier. The authors reported that as the hydration energy of the cations increased in the order of $\mathrm{K}^{+}, \mathrm{Na}^{+}, \mathrm{Li}^{+}, \mathrm{Ca}^{2+}$ and $\mathrm{Mg}^{2+}$, the permeation rate decreased. According to molecular modeling, the barrier for ions to enter graphene capillaries notably increases at interlayer spacings below $9 \AA$. To pass through such a narrow channel, the ion must lose two water molecules, necessitating a substantial energy penalty. On the other hand, the weaker hydrogen bonding between water molecules leads to less hindrance for water to enter and transport through the capillaries. Thus, at a fixed interlayer spacing below $8 \AA$, water is selectively separated from ions.

Low mechanical stability of multilayer graphene membranes. The low mechanical strength and durability of multilayer graphene membranes is a big challenge for their practical application. In this regard, employing potential supports for such membranes has been suggested as an effective approach. For instance, Han et al. ${ }^{22}$ introduced a hybrid 
a

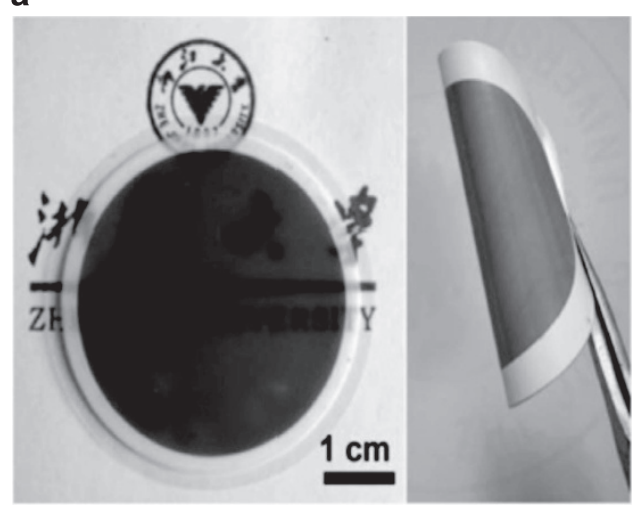

b

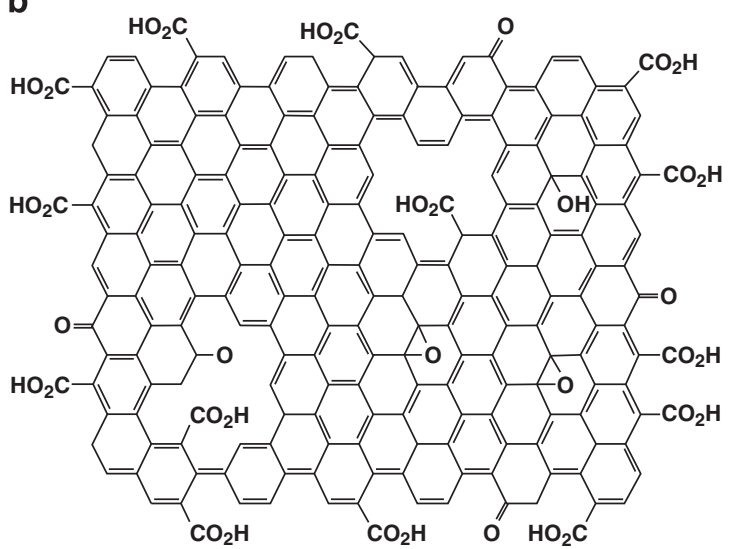

Figure 12 (a) Optical images of ultrathin graphene NF membranes (uGNMs) mounted on an anodic aluminum oxide disk (left) and on a polyvinylidene fluoride membrane (right). (b) Schematic illustrating the chemical structure of base refluxing rGO (brGO), wherein most of the oxidized groups are located on the edges and the periphery of the incorporated holes. (Reprinted with permission from Han et al. ${ }^{22}$ Copyright 2016 John Wiley and Sons.)
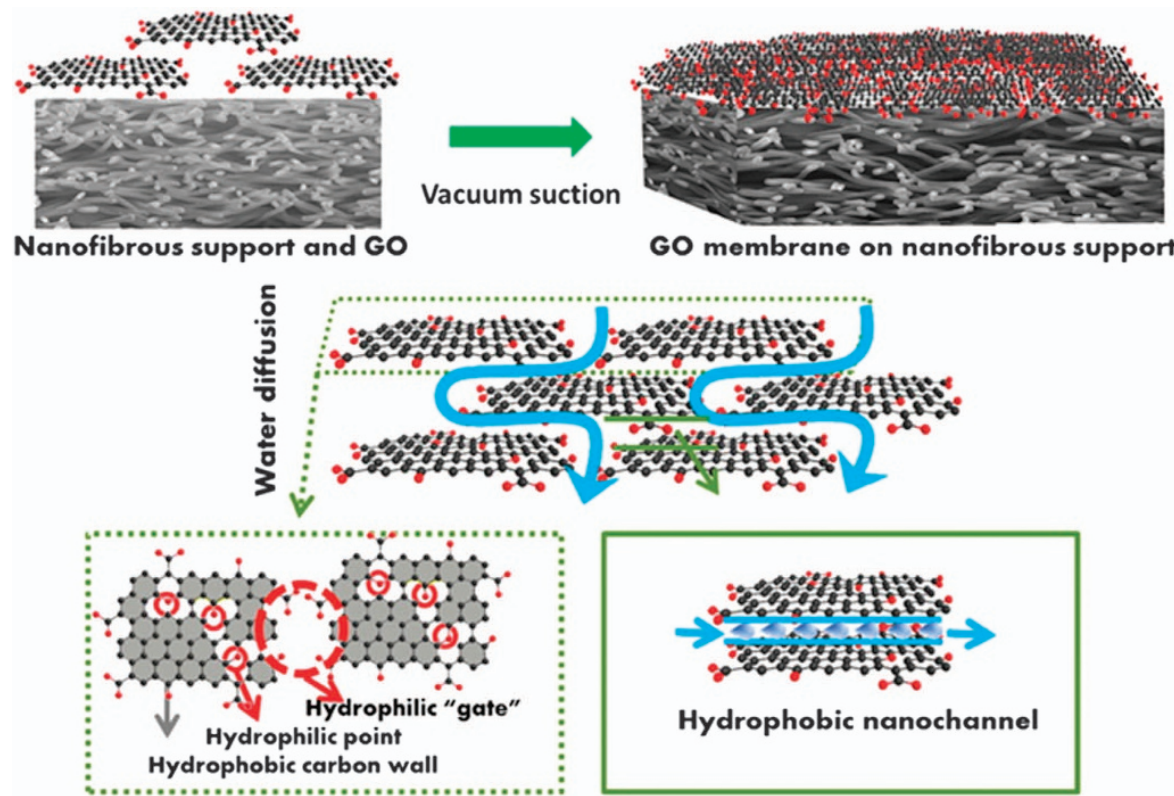

Hydrophobic nanochannel

Figure 13 Schematic illustration of the composite NF membrane, including hydrophilic 'gates' and hydrophobic nanochannels. (Reprinted with permission from Wang et al. ${ }^{79}$ Copyright 2016 American Chemical Society.)

structure composed of ultrathin $(\approx 22-53 \mathrm{~nm}$ thick) graphene NF membranes with $2 \mathrm{D}$ nanochannels deposited on microporous substrates, for example, commercialized microfiltration membranes (of, for example, polyvinylidene fluoride) (Figure 12a). The substrate acts as a support for the ultrathin graphene NF membrane when a pressure up to 20 bar is applied during the NF process. By base refluxing rGO, a well-packed layer membrane was produced via a simple filtrationassisted assembly strategy. The ultrathinness of the membrane resulted in the optimum solvent permeability. Moreover, inherent holes in base refluxing rGO, generated by the oxidation reaction, acted as permeation gates and led to a higher water flux. The water permeability of the ultrathin graphene NF membranes was measured to be as high as $21.81 . \mathrm{m}^{-2} \cdot \mathrm{h}^{-1} \cdot \mathrm{bar}^{-1}$, which is higher than that of commercial NF membranes (for example, the water flux of the commercial low desalting membrane UTC60 is $\left.15.41 . \mathrm{m}^{-2} \cdot \mathrm{h}^{-1} \cdot \mathrm{bar}^{-1}\right) .77$ As seen in Figure $12 \mathrm{~b}$, the residual functional groups on base refluxing $\mathrm{rGO}$ are mainly located at the edges and holes of the graphene sheets. These functional groups not only afford the good dispersibility of base refluxing rGO in water, necessary for creating the well-packed layer structured film, but also contribute to the selectivity of the membrane.

Han et al. ${ }^{22}$ tested their membrane for the retention of several salts, such as $\mathrm{NaCl}, \mathrm{Na}_{2} \mathrm{SO}_{4}, \mathrm{MgCl}_{2}$ and $\mathrm{MgSO}_{4}$, at the concentration of $0.02 \mathrm{M}$ under a pressure of $5 \mathrm{bar}$. The retention sequence of the salt solutions was $\mathrm{R}\left(\mathrm{Na}_{2} \mathrm{SO}_{4}\right)>\mathrm{R}(\mathrm{NaCl})>\mathrm{R}\left(\mathrm{MgSO}_{4}\right)>\mathrm{R}\left(\mathrm{MgCl}_{2}\right)$, which could be explained by the Donnan exclusion theory, which determines the retention performance of charged NF membranes. ${ }^{78}$ Based on this theory, the Donnan potential at the interphase of the solution and membrane excludes co-ions from the membrane. To maintain electroneutrality of the solution on each side of the membrane, counter ions must also be excluded. Thus, the retention of salt depends on the valences of its cationic $\left(\mathrm{Z}^{+}\right)$and anionic $\left(\mathrm{Z}^{-}\right)$species 
a

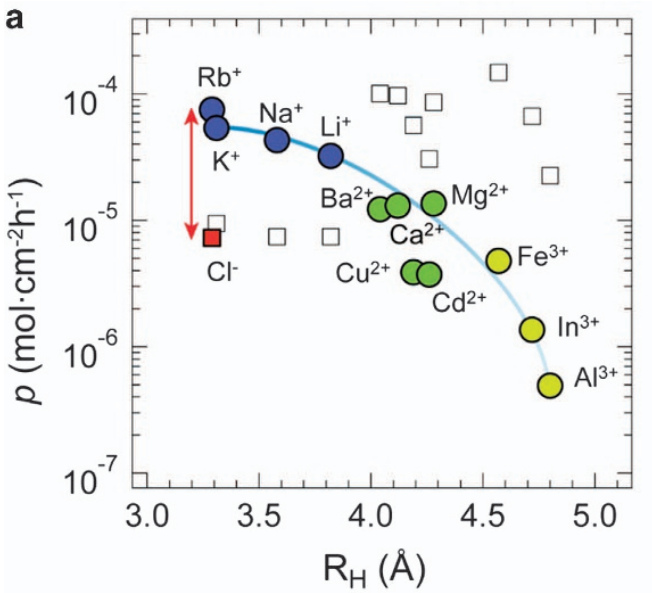

b
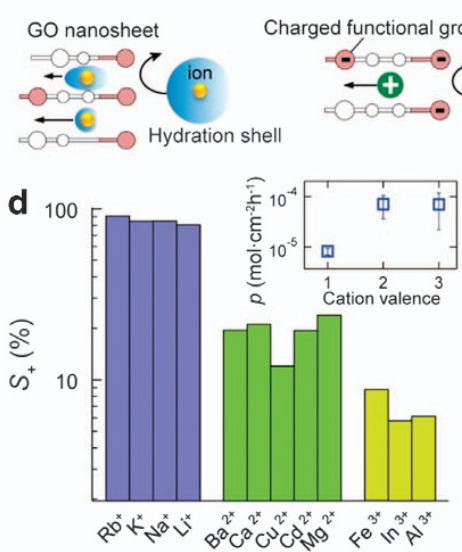

Figure 14 (a) Permeation rates $(p)$ for various cations (circles) and their respective $\mathrm{Cl}^{-}$counterions (open and filled squares) versus the cations' hydrated radius $\left(R_{\mathrm{H}}\right)$. The arrow implies the permeation difference solely by the charge-rejection effects. (b and $\mathbf{c}$ ) Schematic illustrating the ion rejection mechanisms of size exclusion and electrostatic repulsion, respectively. (d) The cationic selectivity of different salts, which approaches over $90 \%$. Inset: the permeation rates of $\mathrm{Cl}^{-}$versus the counterion's valence in the salt, implying the significance of the correlated charge inversion in the subnanometer channels. (Reprinted with permission from Hong et al. ${ }^{84}$ Copyright 2017 American Chemical Society.)

and can be described by the following equation (2): $:^{22}$

$$
R=1-\frac{C_{\mathrm{B}}^{\mathrm{m}}}{C_{\mathrm{B}}}=1-\left(\frac{\left|Z_{\mathrm{B}}\right| C_{\mathrm{B}}}{\left|Z_{\mathrm{B}}\right| C_{\mathrm{B}}^{\mathrm{m}}+C_{\mathrm{X}}^{\mathrm{m}}}\right)^{\left|Z_{\mathrm{B}}\right| /\left|Z_{\mathrm{A}}\right|}
$$

where $Z_{\mathrm{B}}$ and $Z_{\mathrm{A}}$ are the valence of the co-ions and counterions, $C_{\mathrm{B}}$ and $C_{\mathrm{B}} \mathrm{m}$ are the concentrations of the co-ions in solution and in the membrane phase and $C_{X} \mathrm{~m}$ is the membrane charge concentration. The salt retention of this membrane $(20-60 \%)$ is moderate and lower than that of some of commercial desalination NF membranes.

Wang et al. ${ }^{79}$ developed a novel GO-based NF membrane as a composite structure of a GO-selective layer mounted on a polyacrylonitrile nanofibrous mat (Figure 13). Advantageous over traditional support layers, the electrospun nanofibrous mats are highly porous and possess an interconnected pore structure, notably decreasing the membrane mass transfer resistance and thus enhancing the water flux. ${ }^{80}$ GO was chosen as the selective layer due to its ultrathin 2D structure and controllable surface chemistry. The authors deposited GO nanosheets with lateral sizes larger than $200 \mu \mathrm{m}$ on a porous nanofibrous mat by a vacuum suction method. In this structure, GO acts as a barrier, wherein the hydrophobic nanochannels behave as ideal pathways for water molecules between the well-stacked GO nanosheets (Figure 13).

Owing to the unique structure of the GO layer (as thick as $34 \mathrm{~nm}$ ), that is, its approximately $2 \mathrm{D}$ channels surrounded by hydrophobic carbon walls, and the nanofibrous support, a high water flux of $8.21 . \mathrm{m}^{-2} \cdot \mathrm{h}^{-1} \cdot \mathrm{bar}^{-1}$ was obtained. The GO-selective layer mounted on a polyacrylonitrile nanofibrous mat NF membrane $(128 \mathrm{~nm}$ GO thickness) was studied for the rejection of $\mathrm{Na}_{2} \mathrm{SO}_{4}$ and $\mathrm{NaCl}$. The membrane could remove $56.7 \% \mathrm{Na}_{2} \mathrm{SO}_{4}$ and $9.8 \% \mathrm{NaCl}$ through physical sieving and Donnan exclusion mechanisms. Considering that the hydrated radii of $\mathrm{SO}_{4}{ }^{2-}(3.79 \AA), \mathrm{Cl}^{-}(3.32 \AA)$ and $\mathrm{Na}^{+}(3.58 \AA)$ are slightly smaller than that of the carbon nanochannel $(3.98 \AA)$, physical sieving contributes less to salt rejection. As mentioned earlier about the rejection performance of charged NF membranes, the Donnan exclusion mechanism applies here. Considering equation (2), a 1:2 salt (that is, $\mathrm{Na}_{2} \mathrm{SO}_{4}$ ) is rejected with a higher percentage than a 1:1 salt $(\mathrm{NaCl})$. This relationship is the case in this study as well, implying that salts are rejected due to the Donnan effect.
Large-scale production. Another major challenge in the development of multilayer graphene membranes is the scalable liquid film processing techniques able to manufacture large-area membranes. Such methods should also enable the optimum alignment of the GO sheets. Without these facilities, laboratory endeavors are difficult to apply to industrial applications. Akbari et al. ${ }^{81}$ developed a unique approach for the creation of highly ordered, continuous, thin films of stacked GO (discotic nematic phase of GO) on a support membrane. The anisotropic GO nanosheets are readily dispersed in water as stable colloidal suspensions with large volume fractions. By increasing the concentration of anisotropic particles, the orientation entropy of the suspensions decreases. This behavior is compensated by an increase in the translation entropy, whereby a colloidal phase transition occurs from the isotropic to the nematic liquid crystalline phase. ${ }^{82}$ In the liquid crystalline state, the GO sheets become anisotropic. However, they can still flow and react to shear stresses. ${ }^{83}$ To produce concentrated nematic GO dispersions, the authors employed hydrophilic superabsorbent polymer hydrogel beads (typically, a cross-linked polyacrylate-based copolymer). The beads absorb water without dissolving, thus concentrating the GO dispersion. This discotic nematic phase of GO, is used for the creation of large-area GO membranes $\left(13 \times 14 \mathrm{~cm}^{2}\right)$ in a short time of $<5 \mathrm{~s}$ through a shear-induced, industrially adaptable liquid thin film process. The as-prepared membranes are called shear aligned membranes.

The authors compared the NF performance of the shear aligned membrane with that of vacuum filtered membranes and a commercial membrane (NF270 membrane, Dow Chemical Company, Midland, MI, USA). Owing to the formation of organized channels between the GO sheets, the shear aligned membrane had a water permeability of $71 \pm 51 \cdot \mathrm{m}^{-2} \cdot \mathrm{h}^{-1} \cdot \mathrm{MPa}^{-1}$, which is almost seven times higher than that of the vacuum filtration membranes $\left(10 \pm 21 \cdot \mathrm{m}^{-2} \cdot \mathrm{h}^{-1} \cdot \mathrm{MPa}^{-1}\right)$ and almost nine times higher than that of the NF270 membrane. Regarding the retention performance, despite the high retention ( $>90 \%)$ of charged and uncharged solutes with a hydrated radius above $5 \AA$, the membrane showed moderate performance in water desalination and the separation of mono- and divalent salts, including $\mathrm{Na}_{2} \mathrm{SO}_{4}, \mathrm{MgSO}_{4}, \mathrm{MgCl}_{2}$ and $\mathrm{NaCl}$, at a concentration of $2 \mathrm{gl}^{-1}$. The desalination capacity of the membrane was between $30-40 \%$ for all the salts. The desalination mechanisms can be attributed to the small 
interlayer spacing $(\sim 9.5 \AA)$ and the abundant presence of negatively charged oxygen functional groups, such as carboxyl, hydroxyl and epoxy. As mentioned earlier, the negatively charged groups, particularly carboxylic acids, act based on Donnan exclusion theory to repel co-ions and consequently keep counter ions to maintain electroneutrality of the solution on each side of the membrane.

Low desalination capacity. GO multilayer membranes with extraordinary water permeability are favorable candidates for next-generation seawater desalination membranes, if their ion rejection ability can be further enhanced without sacrificing the water flux. In this regard, as shown in Figure 14a, by measuring the diffusion of a diverse range of ions of different sizes and charges (feed salt concentration of $0.1 \mathrm{M}$ ), Hong et al..$^{84}$ demonstrated the various physical mechanisms involved in ionic sieving in GO membranes. These mechanisms include electrostatic repulsion between ions and charged functional groups and compression of the ionic hydration shell within the membrane's nanochannels. In fact, they have shown that ion rejection in such membranes is driven equally by electrostatic repulsion (influenced by the nanochannel surface charge) and activated size exclusion (influenced by the nanochannel height) (Figures $14 \mathrm{~b}$ and c). Accordingly, engineering of the membrane's surface charge can lead to new possibilities for optimizing the total salt rejection without sacrificing the water flux. If so, as shown in Figure 14d, the GO membranes can demonstrate extraordinary charge selectivity as large as $96 \%$, influenced by the negative surface charge of the oxygen-containing functional groups in the membrane's nanochannels. Such possibility holds great promise for the further application of such membranes in the desalination of saline water containing higher salt concentrations.

\section{CONCLUSIONS AND FUTURE WORK}

One of the most serious problems adversely affecting people around the world is insufficient access to clean and potable water and sanitation. This problem will expand in the coming decades as water scarcity begins to occur globally. To address this challenge, extensive research must be conducted to identify advanced novel methods of water purification at lower cost and with less energy. In addition, such technologies must be independent from the use of chemicals and must not impact the environment.

Graphene nanomaterials offer novel solutions for water purification and facilitate the development of advanced water purification membranes, especially for water desalination. Owing to its atomic thickness, assuring high fluid permeability and thus energy/cost efficiency, extraordinary mechanical stability and potential for size-selective transport, graphene is an ideal candidate for future membranes. Graphene-based membranes possess several fascinating advantages over conventional membranes. First, their raw material is graphite, that is, an inexpensive material that affords low membrane fabrication costs. Second, the fabrication procedure of graphene membranes, for example, the fabrication of GO membranes based on GO nanosheets, is quite simple and scalable and enables the technical readiness for scaling up membrane production. This kind of membrane can be further improved by engineering the spacing between the GO layers through the inclusion of different-sized cross-linkers. In addition, the membrane charge and thus the charge-based selectivity can be modified by functionalizing GO with various functional groups.

However, to close the gap between research at the lab scale and practice at the industrial scale, there are still some challenges that must be overcome. First, the selectivity of graphene multilayer membranes is mainly limited to large organic molecules and hydrated ions. Thus, the membranes can perform solely as ultrafiltration or NF membranes. To extend their applicability to desalination, that is, to obtain high salt rejection efficiency, the nanochannel size must be tailored in the subnanometer range. Moreover, if seawater desalination is the target application, realistic salt concentrations and thus solvated ion concentrations should be considered. At the moment, the studied membranes are able to offer the efficient removal of low amounts of salt of a few tens of $\mathrm{mm}$ (versus the average value of $0.6 \mathrm{M}$ for seawater), which is not applicable for large industrial scales. Accordingly, the design and modification of graphene-based membranes should be properly done and, in fact, revolutionized. Considering the high surface area/mass ratio of graphene nanomaterials, this goal could be met by advanced design strategies of, for example, membrane modules exposing large surface areas or by the insertion of negatively charged functional groups, maximizing the electrostatic repulsion with ions. As mentioned earlier, at the moment, the developed graphene membranes can be employed solely as NF membranes, not to reduce seawater salinity to drinking water standards, but to treat mildly brackish feed water. However, by coupling with RO, the membranes can be used in seawater desalination. In addition, future research is required to thoroughly comprehend the transport mechanism of water and solutes in such membranes. In parallel, other potential separation mechanisms (for example, charge and adsorption effects) in addition to sieving must be explored. In this regard, it is necessary to create multifunctional graphene membranes with exceptional antifouling, adsorptive, antimicrobial, and photocatalytic properties. Moreover, for application in environmentally benign and green applications, the long-term stability of graphene membranes in aqueous systems should be improved. This feature can be tracked by ecotoxicological characterizations to guarantee the effective application of graphene membranes and minimize the risks related to possible relevant adverse health and environmental effects. In addition, the mechanical strength and durability of graphene membranes is still a challenge that restricts their practical application. In this regard, employing potential supports for graphene membranes or incorporating them into a host matrix could be a solution. Last but not least, the large-scale, controlled production of graphene membranes for industrialization is still a challenge and needs further study to be realized.

\section{CONFLICT OF INTEREST}

The authors declare no conflict of interest.

\section{ACKNOWLEDGEMENTS}

ME thanks the school of Chemical Engineering at Aalto University, Finland, for providing financial support for his research group.

\section{PUBLISHER'S NOTE}

Springer Nature remains neutral with regard to jurisdictional claims in published maps and institutional affiliations.

1 Shannon, M. A., Bohn, P. W., Elimelech, M., Georgiadis, J. G., Mariñas, B. J. \& Mayes, A. M. Science and technology for water purification in the coming decades. Nature 452, 301-310 (2008).

2 Nicolaï, A., Sumpter, B. G. \& Meunier, V. Tunable water desalination across graphene oxide framework membranes. Phys. Chem. Chem. Phys. 16, 8646-8654 (2014).

3 Homaeigohar, S. \& Elbahri, M. Nanocomposite electrospun nanofiber membranes for environmental remediation. Materials 7, 1017-1045 (2014).

4 Elimelech, M. \& Phillip, W. A. The future of seawater desalination: energy, technology, and the environment. Science 333, 712-717 (2011).

5 Schiermeier, Q. Water: purification with a pinch of salt. Nature 452, 260-261 (2008).

$6 \mathrm{Xu}, \mathrm{K}$., Feng, B., Zhou, C. \& Huang, A. Synthesis of highly stable graphene oxide membranes on polydopamine functionalized supports for seawater desalination. Chem. Eng. Sci. 146, 159-165 (2016). 
7 Semiat, R. Energy issues in desalination processes. Environ. Sci. Technol. 42, 8193-8201 (2008).

8 Fritzmann, C., Löwenberg, J., Wintgens, T. \& Melin, T. State-of-the-art of reverse osmosis desalination. Desalination 216, 1-76 (2007).

9 Van der Bruggen, B., Vandecasteele, C., Van Gestel, T, Doyen, W. \& Leysen, R. A review of pressure-driven membrane processes in wastewater treatment and drinking water production. Environ. Prog. 22, 46-56 (2003).

10 Zheng, Z., Grünker, R. \& Feng, X. Synthetic two-dimensional materials: a new paradigm of membranes for ultimate separation. Adv. Mater. 28, 6529-6545 (2016).

11 Hegab, H. M. \& Zou, L. Graphene oxide-assisted membranes: fabrication and potential applications in desalination and water purification. J. Membr. Sci. 484, 95-106 (2015).

12 Cohen-Tanugi, D. \& Grossman, J. C. Nanoporous graphene as a reverse osmosis membrane: recent insights from theory and simulation. Desalination 366, 59-70 (2015).

13 Lee, K. S. Three ways of linking laboratory endeavours to the realm of policies. Eur. J History Econom. Thought 18, 755-776 (2011).

14 Goh, P. \& Ismail, A. Graphene-based nanomaterial: the state-of-the-art material for cutting edge desalination technology. Desalination 356, 115-128 (2015).

15 Mahmoud, K. A., Mansoor, B., Mansour, A. \& Khraisheh, M. Functional graphene nanosheets: the next generation membranes for water desalination. Desalination 356, 208-225 (2015).

16 Liu, W. W., Chai, S. P., Mohamed, A. R. \& Hashim, U. Synthesis and characterization of graphene and carbon nanotubes: a review on the past and recent developments. J. Ind. Eng. Chem. 20, 1171-1185 (2014).

17 Aghigh, A., Alizadeh, V., Wong, H., Islam, M. S., Amin, N. \& Zaman, M. Recent advances in utilization of graphene for filtration and desalination of water: a review. Desalination 365, 389-397 (2015).

18 An, D., Yang, L., Wang, T. J. \& Liu, B. Separation performance of graphene oxide membrane in aqueous solution. Ind. Eng. Chem. Res. 55, 4803-4810 (2016).

19 Jung, I., Dikin, D. A., Piner, R. D. \& Ruoff, R. S. Tunable electrical conductivity of individual graphene oxide sheets reduced at 'low' temperatures. Nano Lett. 8, 4283-4287 (2008).

20 Balandin, A. A., Ghosh, S., Bao, W., Calizo, I., Teweldebrhan, D., Miao, F \& Lau, C. N. Superior thermal conductivity of single-layer graphene. Nano Lett. 8, 902-907 (2008).

21 Geim, A. K. \& Novoselov, K. S. The rise of graphene. Nat. Mater. 6, 183-191 (2007).

$22 \mathrm{Han}$, Y., Xu, Z. \& Gao, C. Ultrathin graphene nanofiltration membrane for water purification. Adv. Funct. Mater. 23, 3693-3700 (2013).

$23 \mathrm{He}, \mathrm{H}$. , Klinowski, J., Forster, M. \& Lerf, A. A new structural model for graphite oxide. Chem. Phys. Lett. 287, 53-56 (1998).

24 Perreault, F., Fonseca de Faria, A. \& Elimelech, M. Environmental applications of graphene-based nanomaterials. Chem. Soc. Rev. 44, 5861-5896 (2015).

25 Chang, J., Zhou, G., Christensen, E. R., Heideman, R. \& Chen, J. Graphene-based sensors for detection of heavy metals in water: a review. Anal. Bioanal. Chem. 406 3957-3975 (2014).

26 Bunch, J. S., Verbridge, S. S., Alden, J. S., van der Zande, A. M., Parpia, J. M., Craighead, H. G. \& McEuen, P. L. Impermeable atomic membranes from graphene sheets. Nano Lett. 8, 2458-2462 (2008).

27 Berry, V. Impermeability of graphene and its applications. Carbon 62, 1-10 (2013).

28 O'Hern, S. C., Stewart, C. A., Boutilier, M. S., Idrobo, J. C., Bhaviripudi, S., Das, S. K., Kong, J., Laoui, T., Atieh, M. \& Kornik, R. Selective molecular transport through intrinsic defects in a single layer of CVD graphene. ACS Nano 6, 10130-10138 (2012).

29 Lee, K. P., Arnot, T. C. \& Mattia, D. A review of reverse osmosis membrane materials for desalination-development to date and future potential. J. Membr. Sci. 370, 1-22 (2011).

30 Cohen-Tanugi, D. \& Grossman, J. C. Water desalination across nanoporous graphene. Nano Lett. 12, 3602-3608 (2012).

$31 \mathrm{Hu}, \mathrm{M}$. \& Mi, B. Enabling graphene oxide nanosheets as water separation membranes. Environ. Sci. Technol. 47, 3715-3723 (2013).

32 Suk, M. E. \& Aluru, N. R. Molecular and continuum hydrodynamics in graphene nanopores. RSC Adv. 3, 9365-9372 (2013).

33 Thomas, M., Corry, B. \& Hilder, T. A. What have we learnt about the mechanisms of rapid water transport, ion rejection and selectivity in nanopores from molecular simulation? Small 10, 1453-1465 (2014).

34 Corry, B. Designing carbon nanotube membranes for efficient water desalination. J. Phys. Chem. B 112, 1427-1434 (2008).

35 Zhao, S., Xue, J. \& Kang, W. Ion selection of charge-modified large nanopores in a graphene sheet. J. Chem. Phys. 139, 114702 (2013).

$36 \mathrm{He}, \mathrm{Z}$., Zhou, J., Lu, X. \& Corry, B. Bioinspired graphene nanopores with voltage-tunable ion selectivity for $\mathrm{Na}$ + and K+. ACS Nano 7, 10148-10157 (2013).

37 Sint, K., Wang, B. \& Král, P. Selective ion passage through functionalized graphene nanopores. J. Am. Chem. Soc. 130, 16448-16449 (2008).

38 Darden, T., York, D. \& Pedersen, L. Particle mesh Ewald: an N.log $(\mathrm{N})$ method for Ewald sums in large systems. J. Chem. Phys. 98, 10089-10092 (1993).

39 Bae, S., Kim, H., Lee, Y., Xu, X., Park, J. S., Zheng, Y., Balakrishnan, J., Lei, T., Kim, H. R., Song, Y. I., Kim, Y. J., Kim, K. S., Özyilmaz, B., Ahn, J. H., Hong, B. H. \& lijima, S. Roll-to-roll production of 30-inch graphene films for transparent electrodes. Nat. Nanotechnol. 5, 574-578 (2010).

40 Surwade, S. P., Smirnov, S. N., Vlassiouk, I. V., Unocic, R. R., Veith, G. M., Dai, S. \& Mahurin, S. M. Water desalination using nanoporous single-layer graphene. Nat. Nanotechnol. 10, 459-464 (2015).
41 Russo, C. J. \& Golovchenko, J. Atom-by-atom nucleation and growth of graphene nanopores. Proc. Natl Acad. Sci. USA 109, 5953-5957 (2012).

42 Garaj, S., Liu, S., Golovchenko, J. A. \& Branton, D. Molecule-hugging graphene nanopores. Proc. Natl Acad. Sci. USA 110, 12192-12196 (2013).

43 Koenig, S. P., Wang, L., Pellegrino, J. \& Bunch, J. S. Selective molecular sieving through porous graphene. Nat. Nanotechnol. 7, 728-732 (2012).

44 Cheng, Y., Kaloni, T. P., Schwingenschlögl, U. \& Zhu, Z. Oxidation of graphene in ozone under ultraviolet light. Appl. Phys. Lett. 101, 073110 (2012).

45 Wu, S., Yang, R., Shi, D. \& Zhang, G. Identification of structural defects in graphitic materials by gas-phase anisotropic etching. Nanoscale 4, 2005-2009 (2012).

46 Liu, L., Ryu, S., Tomasik, M. R., Stolyarova, E., Jung, N., Hybertsen, M. S., Steigerwald, M. L., Brus, L. E. \& Flynn, G. W. Graphene oxidation: thicknessdependent etching and strong chemical doping. Nano Lett. 8, 1965-1970 (2008).

47 O'Hern, S. C., Boutilier, M. S., Idrobo, J. C., Song, Y., Kong, J., Laoui, T., Atieh, M. \& Karnik, R. Selective ionic transport through tunable subnanometer pores in single-layer graphene membranes. Nano Lett. 14, 1234-1241 (2014).

48 Boutilier, M. S. H., Sun, C., O'Hern, S. C., Au, H., Hadjiconstantinou, N. G. \& Karnik, R. Implications of permeation through intrinsic defects in graphene on the design of defecttolerant membranes for gas separation. ACS Nano 8, 841-849 (2014).

49 O'Hern, S. C., Jang, D., Bose, S., Idrobo, J. C., Song, Y., Laoui, T., Kong, J. \& Karnik, R. Nanofiltration across defect-sealed nanoporous monolayer graphene. Nano Lett. 15, 3254-3260 (2015).

50 Cohen-Tanugi, D., Lin, L. C. \& Grossman, J. C. Multilayer nanoporous graphene membranes for water desalination. Nano Lett. 16, 1027-1033 (2016).

51 Dikin, D. A., Stankovich, S., Zimney, E. J., Piner, R. D., Dommett, G. H. B., Evmenenko, G., Nguyen, S. T. \& Ruoff, R. S. Preparation and characterization of graphene oxide paper. Nature 448, 457-460 (2007).

52 Dreyer, D. R., Park, S., Bielawski, C. W. \& Ruoff, R. S. The chemistry of graphene oxide. Chem. Soc. Rev. 39, 228-240 (2010).

$53 \mathrm{Mi}, \mathrm{B}$. Graphene oxide membranes for ionic and molecular sieving. Science $\mathbf{3 4 3}$ 740-742 (2014).

54 Gin, D. L. \& Noble, R. D. Designing the next generation of chemical separation membranes. Science 332, 674-676 (2011).

55 Joshi, R., Carbone, P., Wang, F., Kravets, V., Su, Y., Grigorieva, I., Wu, H. A., Geim, A. K. \& Nair, R. R. Precise and ultrafast molecular sieving through graphene oxide membranes. Science 343, 752-754 (2014).

56 Nair, R., Wu, H., Jayaram, P., Grigorieva, I. \& Geim, A. Unimpeded permeation of water through helium-leak-tight graphene-based membranes. Science 335, 442-444 (2012).

57 Kannam, S. K., Todd, B., Hansen, J. S. \& Daivis, P. J. Slip length of water on graphene: limitations of non-equilibrium molecular dynamics simulations. J. Chem. Phys. 136, 024705 (2012).

58 Wei, N., Peng, X. \& Xu, Z. Understanding water permeation in graphene oxide membranes. ACS Appl. Mater. Interfaces 6, 5877-5883 (2014).

59 Huang, L., Li, Y., Zhou, Q., Yuan, W. \& Shi, G. Graphene oxide membranes with tunable semipermeability in organic solvents. Adv. Mater. 27, 3797-3802 (2015).

60 Huang, L., Chen, J., Gao, T., Zhang, M., Li, Y., Dai, L., Qu, L. \& Shi, G. Reduced graphene oxide membranes for ultrafast organic solvent nanofiltration. Adv. Mater. 28, 8669-8674 (2016).

61 Buchsteiner, A., Lerf, A. \& Pieper, J. Water dynamics in graphite oxide investigated with neutron scattering. J. Phys. Chem. B 110, 22328-22338 (2006).

62 Eda, G. \& Chhowalla, M. Chemically derived graphene oxide: towards large-area thin-film electronics and optoelectronics. Adv. Mater. 22, 2392-2415 (2010).

63 Wei, N., Lv, C. \& Xu, Z. Wetting of graphene oxide: a molecular dynamics study. Langmuir 30, 3572-3578 (2014).

64 Sun, P., Zhu, M., Wang, K., Zhong, M., Wie, J., Wu, D., Xu, Z. \& Zhu, H. Selective ion penetration of graphene oxide membranes. ACS Nano 7, 428-437 (2013).

65 Sun, P., Zheng, F., Zhu, M., Song, Z., Wang, K., Zhong, M., Wu, D., Little, R. B., Xu, Z. \& Zhu, H. Selective trans-membrane transport of alkali and alkaline earth cations through graphene oxide membranes based on cation $-\pi$ interactions. ACS Nano 8 , 850-859 (2014).

66 Sun, P., Wang, K., Wie, J., Zhong, M., Wu, D. \& Zhu, H. Effective recovery of acids from iron-based electrolytes using graphene oxide membrane filters. J. Mater. Chem. A 2, 7734-7737 (2014)

67 Coleman, M. \& Tang, X. Diffusive transport of two charge equivalent and structurally similar ruthenium complex ions through graphene oxide membranes. Nano Res 8 $1128-1138$ (2015)

68 Huang, H., Mao, Y., Ying, Y., Liu, Y., Sun, L. \& Peng, X. Salt concentration, pH and pressure controlled separation of small molecules through lamellar graphene oxide membranes. Chem. Commun. 49, 5963-5965 (2013).

69 Huang, H., Song, Z., Wie, N., Shi, L., Mao, Y., Ying, Y., Sun, L., Xu, Z. \& Peng, X. Ultrafast viscous water flow through nanostrand-channelled graphene oxide membranes. Nat. Commun. 4, 2979 (2013).

70 Wang, W., Eftekhari, E., Zhu, G., Zhang, X., Yan, Z. \& Li, Q. Graphene oxide membranes with tunable permeability due to embedded carbon dots. Chem. Commun. 50, 13089-13092 (2014)

71 Burress, J. W., Gadipelli, S., Ford, J., Simmons, J. M., Zhou, W. \& Yildirim, T. Graphene oxide framework materials: theoretical predictions and experimental results. Angew. Chem. Int. Ed. 49, 8902-8904 (2010).

72 Huang, L., Zhang, M., Li, C. \& Shi, G. Graphene-based membranes for molecular separation. J. Phys. Chem. Lett. 6, 2806-2815 (2015).

73 Romanos, G., Pastrana-Martínez, L. M., Tsoufis, T., Athanasekou, C., Galata, E., Katsaros, F, Favvas, E., Beltsios, K. G., Siranidi, E., Falaras, P., Psycharis, V. 
\& Silva, A. M. T. A facile approach for the development of fine-tuned self-standing graphene oxide membranes and their gas and vapor separation performance. J. Membr. Sci. 493, 734-747 (2015).

74 Yeh, C. N., Raidongia, K., Shao, J., Yang, Q. H. \& Huang, J. On the origin of the stability of graphene oxide membranes in water. Nat. Chem. 7, 166-170 (2015).

75 Renteria, J. D., Ramirez, S., Malekpour, H., Alonso, B., Centeno, A., Zurutuza, A Cocemasov, A. I., Nika, D. L. \& Balandin, A. A. Strongly anisotropic thermal conductivity of free-standing reduced graphene oxide films annealed at high temperature. Adv. Funct. Mater. 25, 4664-4672 (2015).

76 Abraham, J., Vasu, K. S., Williams, C. D., Gopinadhan, K., Su, Y., Cherian, C. T., Dix, J., Prestat, E., Haigh, S. J., Grigorieva, I. V., Carbone, P., Geim, A. K. \& Nair, R. R. Tunable sieving of ions using graphene oxide membranes. Nat. Nanotechnol. 12, 546-550 (2017).

77 Agenson, K. O., Oh, J. I. \& Urase, T. Retention of a wide variety of organic pollutants by different nanofiltration/reverse osmosis membranes: controlling parameters of process. J. Membr. Sci. 225, 91-103 (2003).

78 Schaep, J., Van der Bruggen, B., Vandecasteele, C. \& Wilms, D. Influence of ion size and charge in nanofiltration. Sep. Purif. Technol. 14, 155-162 (1998).

79 Wang, J., Zhang, P., Liang, B., Liu, Y., Xu, T., Wang, L., Cao, B. \& Pan, K. Graphene oxide as an effective barrier on a porous nanofibrous membrane for water treatment. ACS Appl. Mater. Interfaces 8, 6211-6218 (2016).

80 Homaeigohar, S., Buhr, K. \& Ebert, K. Polyethersulfone electrospun nanofibrous composite membrane for liquid filtration. J. Membr. Sci. 365, 68-77 (2010).

81 Akbari, A., Sheath, P., Martin, S. T., Shinde, D. B., Shaibani, M., Banerjee, P. C., Tkacz, R., Bhattacharyya, D. \& Majumder, M. Large-area graphene-based nanofiltration membranes by shear alignment of discotic nematic liquid crystals of graphene oxide. Nat. Commun. 7, 10891 (2016).

82 Tkacz, R., Oldenbourg, R., Mehta, S. B., Miansari, M., Verma, A. \& Majumder, M. $\mathrm{pH}$ dependent isotropic to nematic phase transitions in graphene oxide dispersions reveal droplet liquid crystalline phases. Chem. Commun. 50, 6668-6671 (2014).

83 Carlsson, T. Remarks on the flow alignment of disc like nematics. J. Phys. 44, 909-911 (1983).

84 Hong, S., Constans, C., Surmani Martins, M. V., Seow, Y. C., Guevara Carrió, J. A. \& Garaj, S. Scalable graphene-based membranes for ionic sieving with ultrahigh charge selectivity. Nano Lett. 17, 728-732 (2017).

85 Lee, A., Elam, J. W. \& Darling, S. B. Membrane materials for water purification: design, development, and application. Environ. Sci: Water Res. Technol. 2, 17-42 (2016).

86 Zhao, J., Wang, Z., White, J. C. \& Xing, B. Graphene in the aquatic environment: adsorption, dispersion, toxicity and transformation. Environ. Sci. Technol. 48, 9995-10009 (2014).

87 Wang, E. N. \& Karnik, R. Water desalination: Graphene cleans up water. Nat. Nanotechnol. 7, 552-554 (2012).

(i) This work is licensed under a Creative Commons Attribution 4.0 International License. The images or other third party material in this article are included in the article's Creative Commons license, unless indicated otherwise in the credit line; if the material is not included under the Creative Commons license, users will need to obtain permission from the license holder to reproduce the material. To view a copy of this license, visit http:// creativecommons.org/licenses/by/4.0/

(C) The Author(s) 2017 